\title{
Macaque Neuron Instance Segmentation only with Point Annotations based on Multiscale Fully Convolutional Regression Neural Network
}

Zhenzhen YOU ( $\nabla$ zhenzhen_you89@163.com )

Xi'an University of Technology https://orcid.org/0000-0003-3882-4737

Ming Jiang

Xidian University

Zhenghao Shi

Xi'an University of Technology

Cheng Shi

Xi'an University of Technology

Shuangli Du

Xi'an University of Technology

Jimin Liang

Xidian University

\section{Anne-Sophie Hérard}

Centre CEA Paris-Saclay - Site de Fontenay-aux-Roses: Commissariat a l'energie atomique et aux energies alternatives Site de Fontenay-aux-Roses

\section{Caroline Jan}

Centre CEA Paris-Saclay - Site de Fontenay-aux-Roses: Commissariat a l'energie atomique et aux energies alternatives Site de Fontenay-aux-Roses

Nicolas Souedet

Centre CEA Paris-Saclay - Site de Fontenay-aux-Roses: Commissariat a l'energie atomique et aux energies alternatives Site de Fontenay-aux-Roses

Thierry Delzescaux

Centre CEA Paris-Saclay - Site de Fontenay-aux-Roses: Commissariat a l'energie atomique et aux energies alternatives Site de Fontenay-aux-Roses

\section{Research Article}

Keywords: Macaque Neuron Instance, Convolutional Regression, Competitive region

Posted Date: February 10th, 2021

DOl: https://doi.org/10.21203/rs.3.rs-181728/v1 
License: (c) (i) This work is licensed under a Creative Commons Attribution 4.0 International License. Read Full License

Version of Record: A version of this preprint was published at Neural Computing and Applications on October 4th, 2021. See the published version at https://doi.org/10.1007/s00521-021-06574-7. 


\title{
Macaque Neuron Instance Segmentation only with Point Annotations based on Multiscale Fully Convolutional Regression Neural Network
}

\author{
Zhenzhen You ${ }^{1,2}$, Ming Jiang ${ }^{3}$, Zhenghao Shi ${ }^{1}$, Cheng Shi ${ }^{1}$, Shuangli Du ${ }^{1}$, Jimin Liang ${ }^{4}$, \\ Anne-Sophie Hérard ${ }^{2}$, Caroline $\mathrm{Jan}^{2}$, Nicolas Souedet ${ }^{2}$, Thierry Delzescaux ${ }^{2}$ \\ ${ }^{1}$ Shaanxi Key Laboratory for Network Computing and Security Technology, School of Computer Science and \\ Engineering, Xi'an University of Technology, Xi'an, China \\ ${ }^{2}$ CEA-CNRS-UMR 9199, MIRCen, Fontenay-aux-Roses, Université Paris-Saclay, France \\ ${ }^{3}$ National Laboratory of Radar Signal Processing, Xidian University, Xi'an, China \\ ${ }^{4}$ School of Electronic Engineering, Xidian University, Xi'an, China
}

\begin{abstract}
In the field of biomedicine, instance segmentation / individualization is important in analyzing the number, the morphology and the distribution of neurons for the whole slide images. Traditionally, biologists apply stereology technique to manually count the number of neurons in the regions of interest and estimate the number in anatomical regions or the entire brain. This is very tedious and time-consuming. In this paper, we propose a multiscale fully convolutional regression neural network combined with competitive region growing technique to individualize size-varying and touching neurons in major anatomical regions of macaque brain. Given that neuron instance or contour annotations are infeasible to obtain in certain regions, such as dentate gyrus where thousands of touching neurons are present, we ask expert to perform point annotations in the center location of neurons (noted as neuron centroids) for training. Thanks to the multiscale resolution achieved by parallel multiple receptive fields and different network depths, our proposed network succeeds in detecting the centroids of size-varying and touching neurons. Competitive region growing is then applied on these centroids to achieve neuron instance segmentation. Experiments on macaque brain data suggest that our proposed method outperform the state-of-the-art methods in terms of neuron instance segmentation performance. To our knowledge, this is the first deep learning research work to individualize size-varying and touching neurons only using point annotations in major anatomical regions in the macaque brain. The cartographic representation of the number and the average orientation of neurons illustrates the synthesis of multi-gigabyte image information in the form of cartography, and shows the characteristics of different anatomical regions from mesoscopic level.
\end{abstract}

\section{Introduction}

Accurate neuron instance segmentation / individualization is important for neuron analysis, providing useful information of neurons, such as the number, the morphology and the distribution of neurons, that helps to study brain development, aging and cytoarchitecture (Andersen et al., 2016; Andrey et al., 2010; Karlsen and Pakkenberg, 2011; Larsen et al., 2006; Pakkenberg and Gundersen, 1997; Pelvig et al., 2008; Walløe et al., 2014; Williams and Herrup, 1988). In pathological brains, for example, it helps to examine neuron loss (Thu et al., 2010; Vicar et al., 2020; Waldvogel et al., 2015). In practice, stereology technique (Gundersen, 1986; Jelsing et al., 2006; West et al., 1991) as the gold standard in the field of neuroscience is used to estimate the number of neurons in anatomical regions. However, this manual operation is cumbersome and time-consuming, and the accuracy of estimation depends on the experts' experience. Moreover, in certain heterogeneous regions, such as hippocampus region, it is difficult to estimate. Therefore, accurate automated methods are requested. So far, a large number of methods have been proposed, but most of them cannot address the individualization problem of large number of size-varying (Figure 1 a1) and touching neurons (Figure 1 a2). Mathematical morphology (Nedzved et al., 2000; Shu et al., 2013) often leads to underindividualization of numerous touching neurons because of the lack of background pixels within the neuron pixels. For instance, in the situation where different sizes of neurons are aggregated, neuron missing or neuron underindividualization problems are often present due to the inappropriate structure element size. Approaches based on concavity detection (Bai et al., 2008; Kothari et al., 2009; Qi, 2014; Riccio et al., 2019; Zhang et al., 2013) cannot give accurate individualization results either, because noise present in neuron contours hamper the computation of clear concavity points. Graph cut methods (Al-Kofahi et al., 2010; Daněk et al., 2009; He et al., 2015; Lou et al., 2012) often cause problems over- and under-individualization problems since the relation between inter-pixels and intra-pixels in numerous touching neurons is difficult to investigate. Region growing methods (Adams and Bischof, 1994; Zucker, 
1976) can correctly separate touching cells if seeds are correctly defined. Watershed algorithm (Cousty et al., 2009; Yang et al., 2006) is often used to separate neurons, but it can also lead to over- and under-individualization due to the noise. In the past years, the emergence of deep learning techniques has led to several applications, such as cell counting (Xie et al., 2016; You et al., 2020) and segmentation (Li et al., 2019; Yoo et al., 2019) from histology sections. Since it is almost impossible to obtain highly accurate artificial labels in the field of cell segmentation, researchers tend to use point annotations for the cell training. Xie et al. (2016) proposed FCRN (Fully Convolutional Regression Network) to regress a cell spatial density map to detect cell centroids, but in roughly rounded cell clump, this method cannot give correct detection results. Yoo et al. (2019) proposed PseudoEdgeNet to guide the network recognize nuclei edges with point annotations. However, the nuclei edge information used for training is not optimal for numerous touching neurons. The examples displayed in their paper concerned merely single individual nuclei. Li et al. (2019) proposed a weakly supervised method for mitosis detection based on expansion of point annotations. They used FCN (Long et al., 2015) to extract mitosis features and designed a concentric loss function which considers mitosis pixels inside a disk with a radius $r$ and background pixels outside a disk with radius $R(R>r)$ for training. But this method cannot separate touching cells and tuning $r$ and $R$ is difficult for neurons with various sizes. U-net (Falk et al., 2019; Ronneberger et al., 2015), U-net++ (Zhou et al., 2020) can be applied for cell counting, detection and segmentation, but with only point annotations, their accuracies are limited in the case of size-varying and large number of touching neurons.

In this paper, we propose a new image processing protocol which aims at automatically separating size-varying and touching neurons. First, the point annotations are in practice disks with a predefined radius in the center of neurons, as shown in Figure 1 b1 \& b2. They are then processed by a Gaussian filter to obtain a probability map which describes the probability of pixels being neuron centroids. This map is used as ground truth to train the neural network, as shown in Figure $1 \mathrm{c} 1 \& \mathrm{c} 2$. The higher the intensity of a pixel in the probability map, the more probable it is the neuron centroid. Then, the predicted probability map of neuron centroids is obtained by applying the proposed multiscale fully convolutional regression neural network. Next, a post-processing step with a series of mathematical morphology operations (erosion, reconstruction, closing) are applied to locate neuron centroids. Finally, based on the neuron centroids, competitive region growing method proposed by You et al. (2019) is applied to obtain neuron individualization results. We applied our method on major anatomical regions in macaque brain. The results demonstrate that our method outperform the state-of-the-art methods, such as FCRN (Xie et al., 2016), U-net (Ronneberger et al., 2015), U-net++ (Zhou et al., 2020) and multiscale CNN (You et al., 2020).
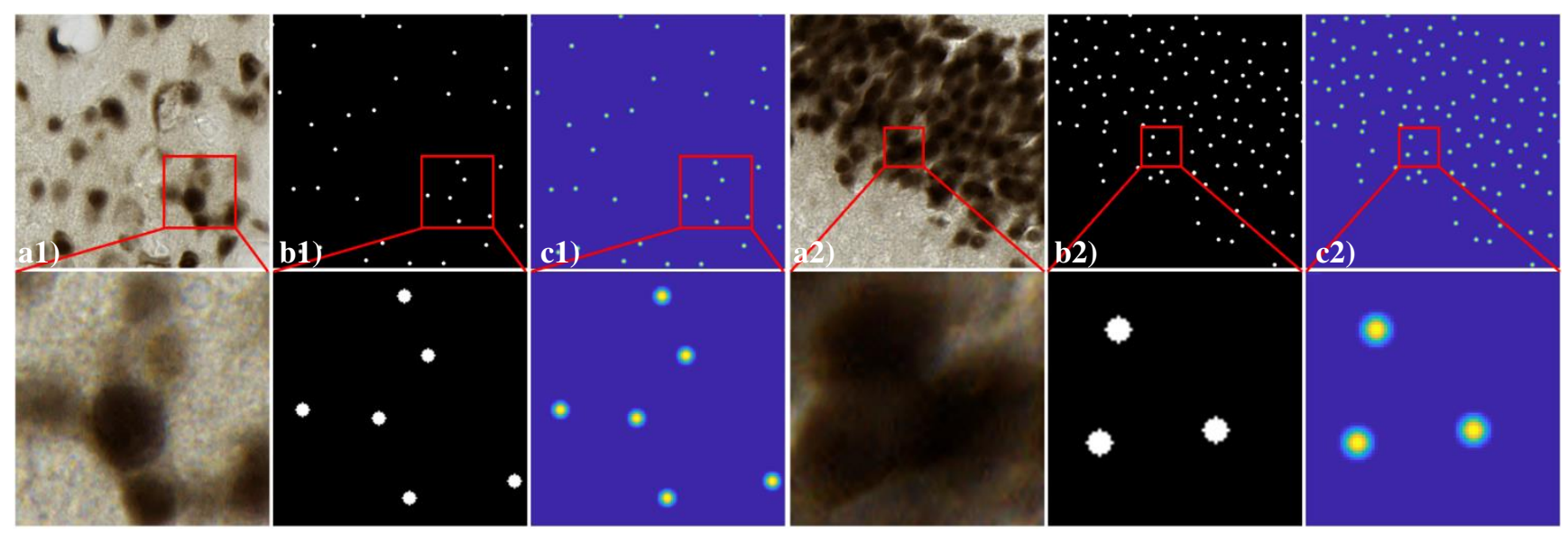

Figure 1. Example images of size-varying and touching neurons. a1 \& a2) Example original images of cortex and hippocampus dentate gyrus. b1 \& b2) Expert annotations (disks with a radius of 5 pixels in center location of neurons) on images of a1 \& a2). c1 \& c2) Preprocessing on expert annotations using a Gaussian filter ( $\sigma=3$ pixels).

\section{Related work}

\subsection{NeuN-stained neurons}

NeuN expression is observed in most neuronal cell types throughout the nervous system (Mullen et al., 1992). An image of NeuN-stained neurons in the cortex of a macaque brain is shown in Figure 2a and the contours based on 
intensity of this image is shown in Figure 2b. Since the distribution of neuron pixel intensity is similar to the Gaussian distribution, a Gaussian filter is used for denoising (Figure 2c). It can be clearly seen that the neurons show obvious dark areas in their center positions. Meanwhile, the pixel intensity varies from high to low as from the edge of the neuron to its center. This kind of images allows to studying neuron number, morphology and distribution from microscopic level using macaque brain which is a model closer to the human brain compared to murine models (Passingham, 2009). In order to perform this study, neuron individualization is crucial and numerous algorithms have been proposed, mainly based on unsupervised segmentation methods, the fully supervised and weakly supervised learning methods.

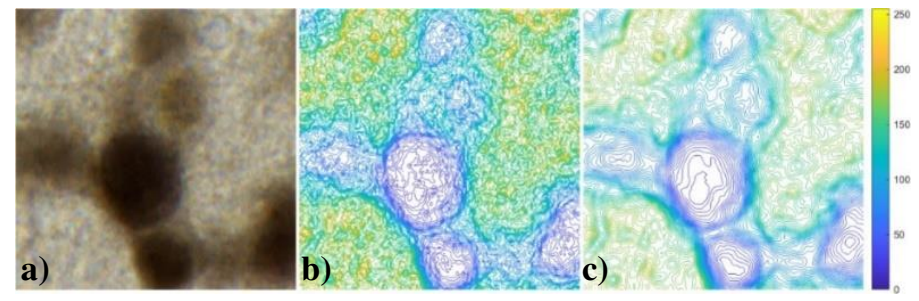

Figure 2. Characteristics of NeuN-stained neurons. a) Original color image in the cortex. b) Contour image based on pixel intensity of a). c) Denoising image of $b$ ) using a Gaussian filter ( $\sigma=3$ pixels).

\subsection{Unsupervised cell segmentation methods}

Unsupervised methods perform instance segmentation through analyzing cell features (intensity, shape, texture, etc.). $\mathrm{He}$ et al. (2015) proposed to segment touching cells based on the features including cell size, spatial location, intervening contours and concavity detection. Zhang et al. (2016) used the spatial relationship of the non-overlapping and overlapping areas as well as Overlapping Translucency Light Transmission Model to segment overlapping cervical cells. Ma et al. (2018) proposed a saliency-based method to detect oligodendrocyte progenitor cells and then used a marker-controlled watershed algorithm to segment them. You et al. (2019) applied a multi-scale series of Gaussian filter for denoising and then performed neuron instance segmentation by applying min-max filter and competitive region growing. Through analyzing Dice values among a series of results, they selected the optimal Gaussian filter and reiterated the instance segmentation.

\subsection{Fully supervised learning-based methods}

For highly accurate cell segmentation, fully-supervised learning-based methods are the most important methods. Unet is a classic method that once achieved top ranks in biomedical data-analysis benchmarks (Falk et al., 2019; Ronneberger et al., 2015; Ulman et al., 2017). The network can learn the pixels relies on cell instance segmentation marked by expert or contour pixels relies on cell outlines drawn by expert. Due to its classic U-shaped and skip connection design, numerous methods have been derived. Rad et al. (2018) proposed a multi-resolution ensemble of stacked dilated U-net for inner cell mass segmentation. Vuola et al. (2019) performed nuclei segmentation which combines the predictions of an ensemble model of U-net and Mask R-CNN. Huang et al. (2019) used U-net and improved level-set to segment overlapped cervical smear cells. There are also studies which learn cell characteristics from the perspective of transformation of ground truth. Naylor et al. (2019) address the cell segmentation as a regression task of the distance map, calculated for each foreground pixel as the chessboard distance to the closest background pixel. However, these methods require cell instance segmentation masks or cell contour masks for training which is time-consuming and labor-intensive work. Moreover, in the region where cells are highly aggregated, experts cannot identify cell instance or contours.

\subsection{Weakly supervised learning-based methods}

The staining of cells often shows a certain pattern, such as neurons, the intensity of NeuN staining gradually increases from the center location of the neuron to the edge. Therefore, experts can relatively easily mark the center location of neurons, i.e. neuron centroids. In the case of having centroid markers, researchers tried weakly supervised learningbased methods for cell segmentation. Li et al. (2019) expanded the weak label of mitosis centroid to a novel label with concentric circles and designed a concentric loss to train the network to perform mitosis segmentation. The architecture proposed by Yoo et al. (2019) was composed of a segmentation network (FPN (Lin et al., 2017) + ResNet-50 (He et al., 2016)) and PseudoEdgeNet. The former was used to localize nuclei as blobs and the latter was used to extract fine 
boundary information without edge annotations by applying four convolution layers and an attention module of an FPN with a Resnet-18. The methods mentioned above are in fact more semantic segmentation than instance segmentation. It is in fact very difficult to achieve cell instance segmentation using weakly supervised learning-based methods, so there are not many methods. However, methods based on weakly supervised learning (Brabandere et al., 2017; Sofiiuk et al., 2019; Wang et al., 2020) in the domain of natural image processing can be used for reference in the domain of medical image processing to achieve cell instance segmentation.

\subsection{Cell detection with point annotations}

Instead of directly performing cell instance segmentation using point annotations, researches from the point of view of cell detection can be first conducted. Xue et al. (2017) applied CNN to regress a fixed length vector which was encoded by a random Gaussian matrix and a k-sparse signal generated based on cell locations from a microscopy image. Cell locations were then recovered through $L_{1}$ minimization / compressed sensing theory. Kainz et al. (2015), Xie et al. (2016), Raza et al. (2019) and You et al. (2020) regard cell detection as a regression problem. The former one uses Random Forest to regress a function of the distance to the center of the closest cell. The latter three design respectively a FCRN, a MapDe and a multiscale CNN to regress cell spatial density / heat map. They then identify cell centers by calculating the local extrema or performing deconvolution operation in the generated score map. With the detected cell centroids, methods like region growing can be applied to achieve cell instance segmentation.

\section{Materials and methods}

\subsection{Biological datasets}

NeuN-stained 40- $\mu$ m-thick serial coronal sections of a healthy macaque brain were digitized using an AxioScan.Z1 (Zeiss) with an in-plane resolution of $0.22 \mu \mathrm{m} /$ pixel ( $\times 20$ magnification). Three datasets from You et al. (2019) were used in this study: 1) segmentation dataset, 2) individualization dataset, and 3) stereology dataset. The segmentation dataset is used to obtain neuron mask that can stand up to staining intensity difference existing in various brain sections. The individualization dataset is used to learn to detect neuron centroids and perform neuron individualization. In order to use deep learning methods, each image of size $5000 \times 5000$ pixels in the individualization dataset was cropped to 144 small images of size $512 \times 512$ pixels as shown in Figure 3. There are 104 overlapped pixels between each two adjacent images in both horizontal and vertical directions. Fifty images of the individualization dataset were then cropped to a total of 7200 images. In this work, a first training data of 152 representative images (i.e. about $2 \%$ of the individualization dataset) from different anatomical regions were used to train the deep learning network and 50 images reconstructed from the 7200 predicted images were used to validate the network. Each neuron was identified by an expert by manually marking a disk ( 5 pixels in radius) in practice in its center location whose intensity is the lowest (darkest) among its neighbors, as shown in Figure $1 \mathrm{~b} 1 \& \mathrm{~b} 2$. The stereology dataset is used to generate cartography of features which reflect neuron morphology and distribution in different anatomical regions from mesoscopic level.

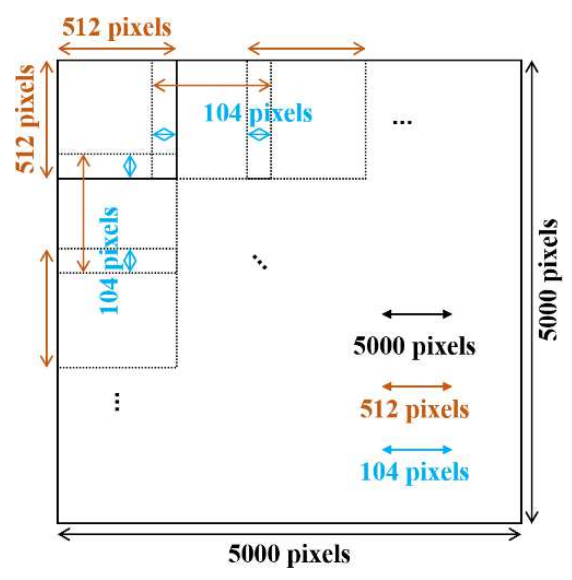

Figure 3. Image cropping. Each image of size $5000 \times 5000$ pixels from individualization dataset from You et al. (2019) was cropped to 144 small images of size $512 \times 512$ pixels adapted for deep learning. There are 104 pixels overlapped between each two adjacent images in both horizontal and vertical direction. 


\subsection{Preprocessing of point annotations}

Expert manually marked a disk (5 pixels in radius) in the center of neurons as shown in Figure 1 b1 \& b2. In order to obtain more accurate detection results, the expert annotations were preprocessed by applying a Gaussian filter $(\sigma=3$ pixels). A probability map was then obtained describing the probability of a pixel being the centroid, as shown in Figure $1 \mathrm{c} 1 \& \mathrm{c} 2$. These images are used as the ground truth of the proposed network. The input of the proposed network is the normalized grayscale images converted from the original color images, calculated as follows (Cheng et al., 2001):

$$
I=(0.299 R+0.587 G+0.114 B) / 255
$$

where $I$ is the input of the proposed network, $R, G$ and $B$ are respectively the red, green and blue channel of the original image.

\subsection{Neuron centroids prediction}

The neuron diameter is between 5 and $30 \mu \mathrm{m}$ (i.e. between 22 and 137 pixels with resolution of $0.22 \mu \mathrm{m} / \mathrm{pixel}$ ) (Andersen et al., 2016). By using the classic U-net or its derivatives with deeper layers, neurons of various sizes can be detected. However, as layer of the network deepens, the features of small-sized neurons gradually disappear. Especially for a large number of aggregated small-sized neurons that are usually present in the region of dentate gyrus, a network with deeper layers will lead to numerous missed detections of such neurons. Research on the automated detection of highly aggregated neurons can effectively capture the features of small-sized neurons (You et al., 2020). Therefore, the module that learn the features of small-sized neurons through parallel multiple receptive fields are integrated into the shallow structure of deep learning model. In addition, using different decoders to the encoders in different network depths favorize supervision of different sizes of neurons. This motivates us to propose a network with parallel multiple receptive fields, optimal depth layer and multiple decoders to detect single individual neurons, size-varying and touching neurons.

Figure 4 presents the architecture of the proposed network which is composed of convolution, ReLU (Rectified Linear Unit), max pooling, and sigmoid function. All the kernels used for convolution operations are of size $1 \times 1,2 \times$ 2 or $3 \times 3$ pixels. Lxy represent layers of different depths where $x$ represents the layer along the vertical direction and $y$ represents the layer along the horizontal direction.

The proposed network consists of two parts: the parallel multiscale encoder network and decoder network. L11 and L21 are the modules of parallel multiple receptive fields to better learn features of numerous aggregated neurons which are inspired from You et al. (2020). The path L11 $\rightarrow$ L21 $\rightarrow$ L31 $\rightarrow$ L22 $\rightarrow$ L13 with skip connections can supervise neuron features in 17 receptive fields of sizes from 1 to 5 pixels in steps of 1 pixel, from 6 to 14 pixels and from 20 to 32 pixels in steps of 2 pixels. As parallel multiple receptive field modules are constructed in the shallow structure, the deep structures are affected and also contain parallel multiple receptive field modules. The path L11 $\rightarrow$ L21 $\rightarrow$ L31 $\rightarrow$ L41 $\rightarrow$ L32 $\rightarrow$ L23 $\rightarrow$ L14 with skip connections supervises larger neuron features in 7 more receptive field of sizes from 56 to 68 pixels in steps of 2 pixels. The path L11 $\rightarrow$ L21 $\rightarrow$ L31 $\rightarrow$ L41 $\rightarrow$ L51 $\rightarrow$ L42 $\rightarrow$ L33 $\rightarrow$ L24 $\rightarrow$ L15 with skip connections supervises even larger neuron features in 7 more receptive field of sizes from 128 to 140 pixels in steps of 2 pixels. The path L11 $\rightarrow$ L21 $\rightarrow$ L31 $\rightarrow$ L41 $\rightarrow$ L51 $\rightarrow$ L61 $\rightarrow$ L52 $\rightarrow$ L43 $\rightarrow$ L34 $\rightarrow$ L25 $\rightarrow$ L16 with skip connections supervises the largest neuron features in 7 more receptive field of sizes from 272 to 284 pixels in steps of 2 pixels. The maximum size of receptive field is more than twice of the largest neuron which helps to supervise the relationship between adjacent touching neurons.

This network was trained by combining four separate loss functions of four paths. We applied a sigmoid function to the outputs (i.e. L13, L14, L15 and L16) from the 4 paths. The detection loss was defined as their sum (equation

(2)). At the inference phase, the prediction from the 4 paths was averaged. Once the prediction results of 7200 images were obtained, we reconstructed 50 images of size $5000 \times 5000$ pixels from corresponding 144 images of size $512 \times$ 512 pixels by summing the prediction maps in the corresponding pixel positions (i.e. reverse process of Figure 3 ).

$$
\mathcal{L}=-\frac{1}{N} \sum_{i=1}^{4} \sum_{n=1}^{N} y_{i, n} \log p_{i, n}
$$

where $y_{i, n}$ is the target labels and $p_{i, n}$ is the predicted probability for the $n^{\text {th }}$ pixel being neuron centroid class in the $i^{\text {th }}$ path, $N$ indicates the number of pixels in one batch. 


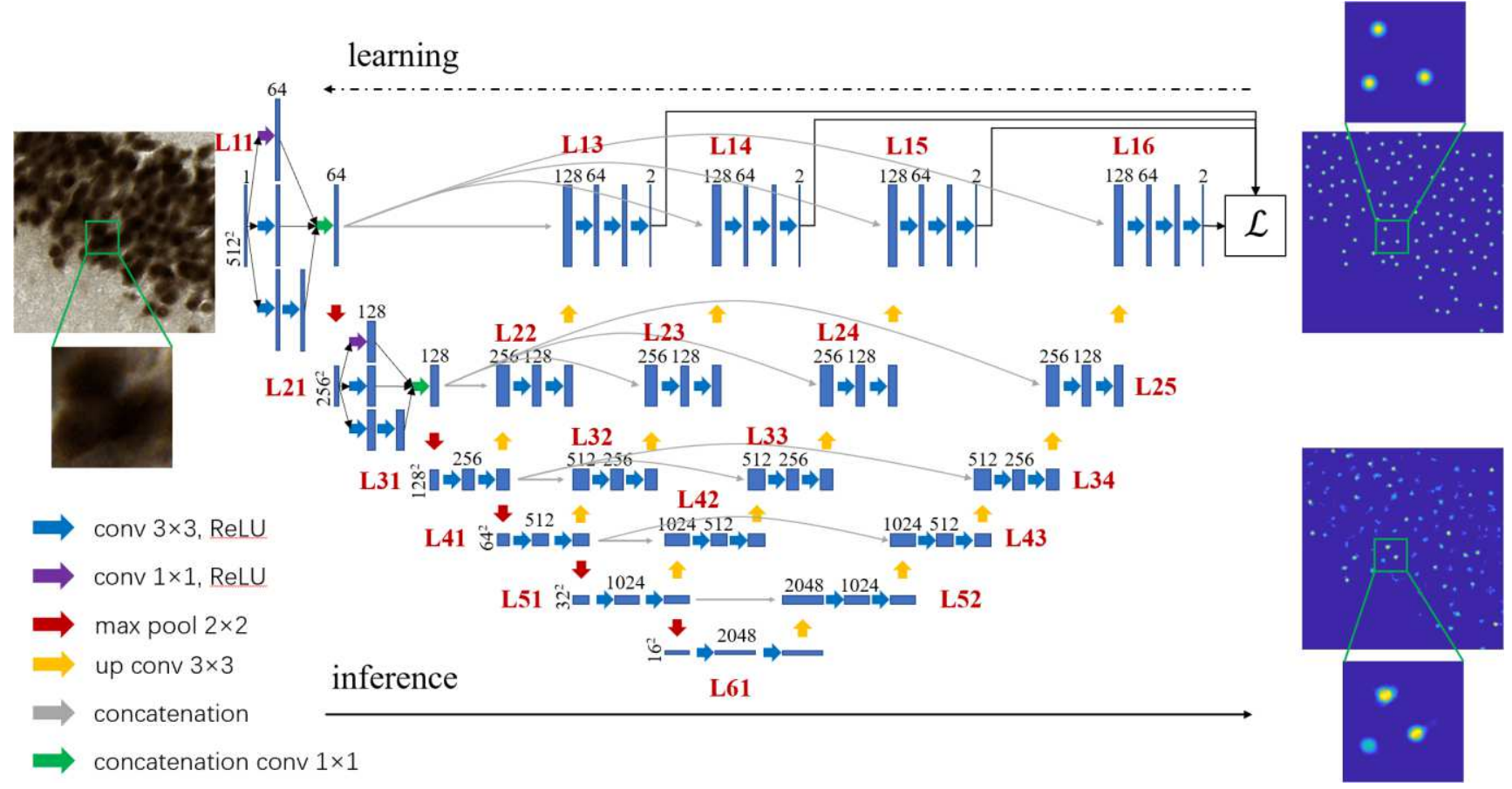

Figure 4. Network structure for neuron centroid detection. The red figures noted as Lxy are the different layers. $x$ represents the layer along the vertical direction and $y$ represents the layer along the horizontal direction. The size of input and output images is $512 \times 512$ pixels. Arrows of different colors represent different operations as described at the left bottom of the figure. The size of images is denoted at the left bottom of the boxes and the number of channels is denoted at the top of the boxes. If there is nothing at the left bottom or on the top, it means the same size of images or the same number of channels as that of previous box.

\subsection{Neuron individualization}

Based on the segmentation dataset, the U-net model was generated (the learning rate was set to 1e-4, Adam optimizer was selected, cross entropy was chosen for the loss function and 300 epochs was performed for training). Neuron mask of the individualization dataset noted $I_{\mathrm{m}}$ (Figure $5 \mathrm{~b}$ ) was then obtained by using this U-net model. On the probability map (Figure $5 \mathrm{c}$ ) predicted by the proposed network, pixels with intensity greater than 0 were extracted as neuron centroid candidates, noted $I_{\mathrm{p}}$ (Figure $5 \mathrm{~d}$ ). Since numerous small receptive fields were applied in the proposed network, mathematical operation should be used to reduce the noise. $I_{\mathrm{p}}$ was first eroded by using a structuring element with 5 pixels in radius. Followed by erosion, reconstruction and closing operation, the sparse pixels were removed (Figure 5 e). In order to remove all the non-neuronal class pixels, $I_{\mathrm{p}}$ was masked by $I_{\mathrm{m}}$ (Figure $5 \mathrm{f}$ ), and then neuron centroids can be calculated as the mass center of connected component (Figure $5 \mathrm{~g}$ ). Neuron contours were then initialized based on these centroids and neurons were separated by applying competitive region growing model (You et al. (2019)).

\subsection{Evaluation metrics}

The accuracy of neuron counting was evaluated by using the relative error $\varepsilon$ which is defend as follows:

$$
\varepsilon=\frac{\left|N_{a}-N_{e}\right|}{N_{e}}
$$

where $N_{\mathrm{a}}$ is the number of neurons detected by the automated method and $N_{\mathrm{e}}$ is the number of neurons marked by expert.

The smaller the relative error, the better the performance of the automated method.

In addition, another criterion considering the position of the individualized neurons and expert centroids was applied. For each individualized neuron, the number of expert centroids contained in the neuron is computed. If exactly one expert centroid is contained, this neuron is considered as correctly individualized. Else, it is either over-individualized (zeros expert centroids) or under-individualized (more than 1 expert centroids, considered as 1 correctly individualized 


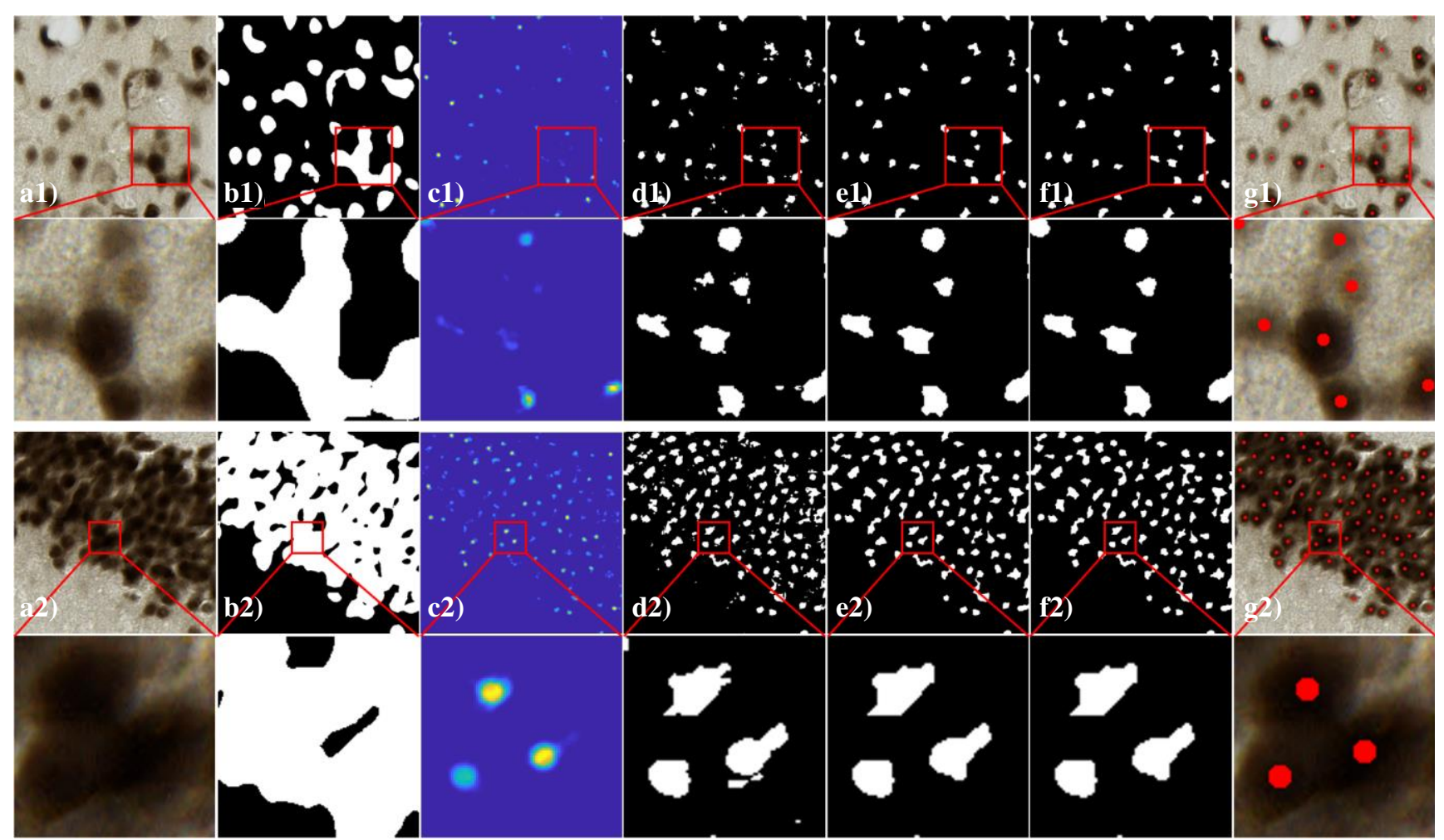

Figure 5. Postprocessing of predicted probability map. a1) \& a2) Example original images of cortex and hippocampus dentate gyrus. b1) \& b2) Neuron mask $I_{\mathrm{m}}$. c1) \& c2) Probability map obtained by using the proposed network. d1) \& d2) Image $I_{\mathrm{p}}$ calculated from c1\& c2) on which the intensity is greater than 0. e1) \& e2) Denoised image based on d1) \& d2) after erosion, reconstruction and closing operations. f1) \& f2) Images of e1) \& e2) masked by $I_{\mathrm{m}}$. g1) \& g2) Original images overlapped with centroids, represented by red disks, calculated as mass center of connected component on f1) \& f2).

thanks to one-to-one correspondence). Recall (R), Precision (P) and F-score (F) are defined as follows:

$$
R=\frac{N_{t}}{N_{e}} ; \quad P=\frac{N_{t}}{N_{a}} ; \quad F=2 \frac{R \times P}{R+P}
$$

where $N_{\mathrm{t}}$ is the number of correctly individualized neurons, $N_{\mathrm{e}}$ and $N_{\mathrm{a}}$ is respectively the number of neurons annotated by expert and computed by automated method.

The larger the value of F-score is, the better the performance of the automated method is.

\subsection{Calculation of descriptor parameters of neurons and generation of cartography of features}

A macaque brain contains millions of neurons of different sizes, shapes, distribution, etc. They can be characterized by a large number of descriptors which can be colorimetric but also morphological. We studied a set of descriptors which can be grouped in the form of several families of descriptors:

- Colorimetric: average and standard deviation of the components of several colorimetric spaces (RGB, HSV, etc.).

- Morphological: mass center, area, perimeter, mean radius, orientation of neurons, etc.

This information is saved in the form of a "neuron vector" of size $n \times m$ ( $n$ being the number of individualized neurons and $m$ being the number of descriptors calculated for each neuron). It can be used to quantify neuron features. The generation of a cartography can be seen as isotropic aggregation in $X$ and $Y$ direction from the original microscopic resolution. When we generate cartographies at mesoscopic level with an aggregation faction of $p \times q$ (aggregation of $p$ pixels in $X$ and $q$ pixels in $Y$ ) from original image of microscopic level, each pixel in the cartography synthesizes the value of a descriptor of the population of neurons if their mass centers (saved in the "neuron vector") after aggregation are located in that pixel. Figure 6 displays the flowchart of the cartography calculation of the hippocampus of macaque. 

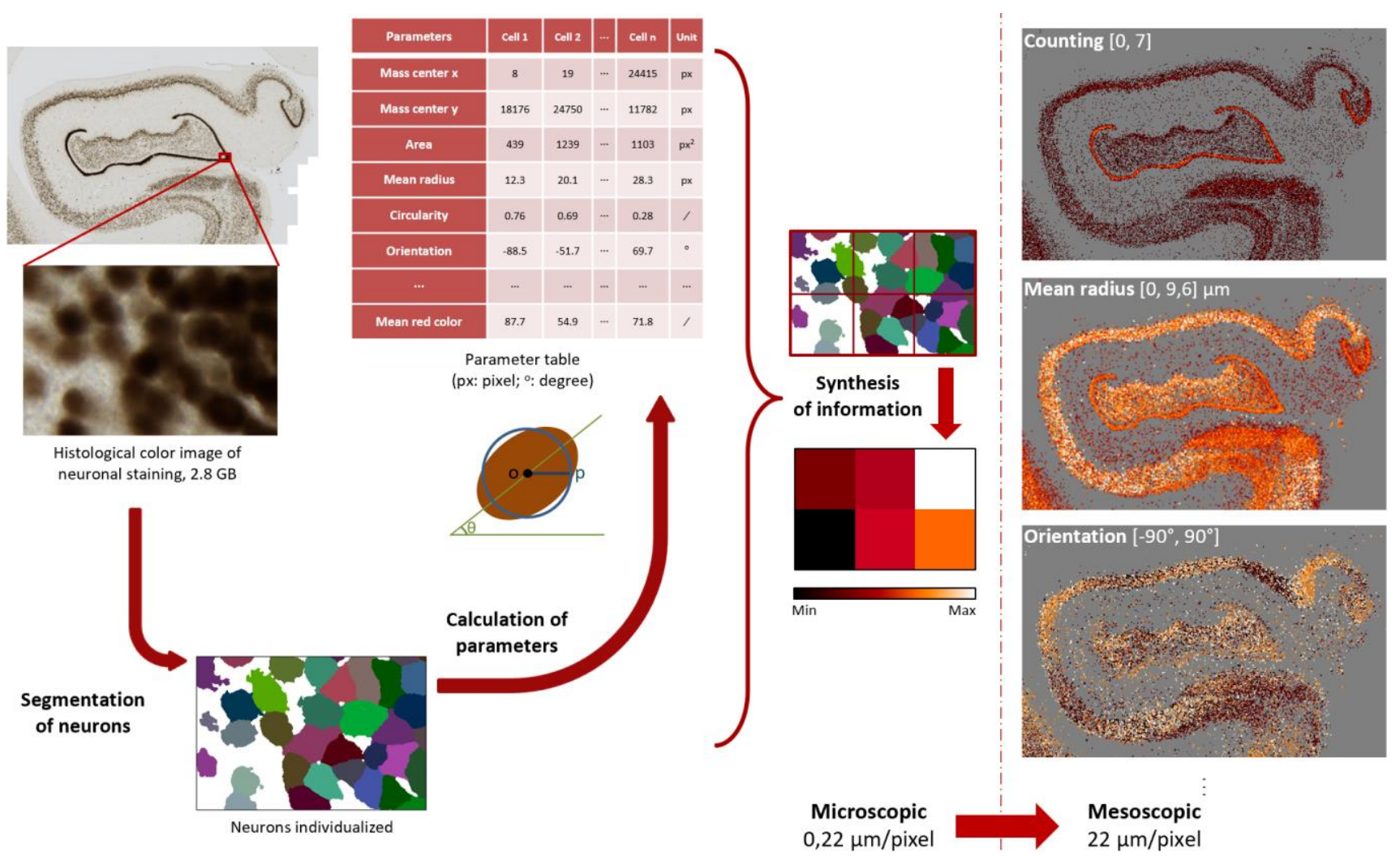

Figure 6. Strategy to generate features cartographies of hippocampus at mesoscopic scale.

\section{Results}

\subsection{Implementation}

About the parameters used in the proposed network, the learning rate was set to 1e-5, Adam optimizer was selected and cross entropy was chosen for the loss function. The proposed network was trained on a computer with a NVIDIA GeForce RTX 2080 Ti GPU with 11GB memory. It took about $10 \mathrm{~h}$ when performing 500 epochs for training.

\subsection{Qualitative visual inspection}

We respectively applied the proposed network, unsupervised method proposed by You et al. (2019), deep learning methods of U-net (Ronneberger et al., 2015), U-net++ (Zhou et al., 2020), FCRN (Xie et al., 2016) and multiscale CNN (You et al., 2020) to obtain the prediction map of centroid. The final individualization results were calculated using the same postprocessing protocol as described in section 3.4.

Figure 7 shows typical results obtained on 9 representative images by applying different automated methods. From the $1^{\text {st }}$ row to the $9^{\text {th }}$, the images are obtained in the anatomical region of caudate, claustrum, cortex, hippocampus CA1 (cornu ammonis), hippocampus CA3, hippocampus dentate gyrus, putamen, subiculum and thalamus, respectively. Red points in the $1^{\text {st }}$ column overlapped with original color images are expert annotations of neuron centroids. It is obvious that the neuron characteristics in various anatomical regions are different. Figure 7 a1 represents simple images with a few isolated neurons of different sizes in caudate. Figure 7 a2 represents some elongated neurons in claustrum. Neurons in Figure 7 a 3 from cortex are generally bigger than those in caudate and claustrum. Figure 7 a $4 \&$ a5 represent two different subregions of hippocampus in which neuron expressions are different from normal NeuN-stained neurons (darker in center and lighter in edge). Figure 7 a6 represents an extremely complex case where large numbers of neurons are aggregated and their edges are difficult to distinguish. Neurons in Figure 7 a7 \& a8 are distributed sparse and relatively small. Moreover, the staining of neurons in Figure 7 a7 is lighter than that in Figure 7 a8. Neurons in Figure 7 a9 are distributed sparser and are with a wide range of sizes.

The probability maps of candidate pixels of neuron centroid obtained by the proposed network are shown in 


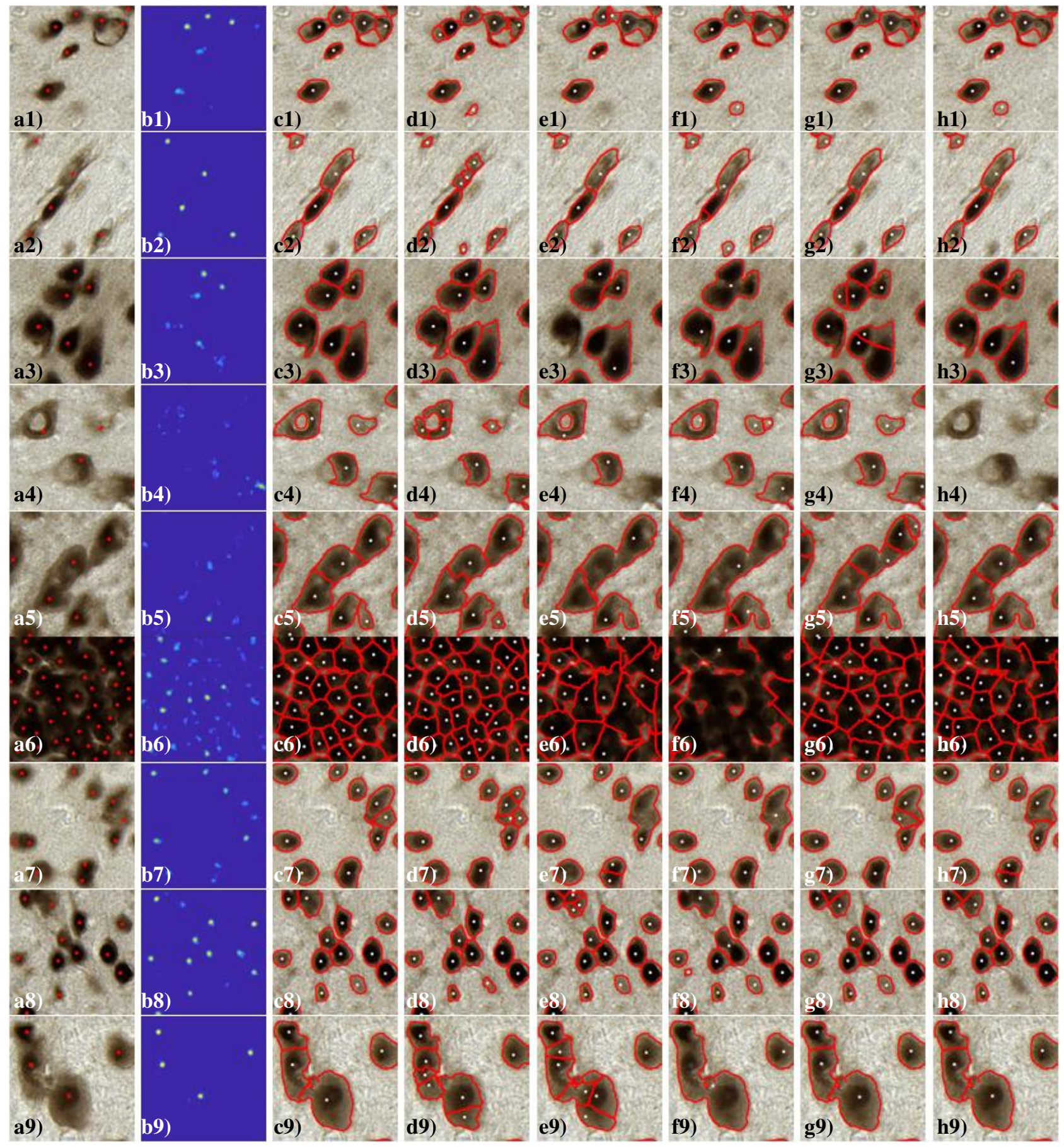

Figure 7. Comparison results of neuron centroid detection and neuron individualization obtained on images from different anatomical regions. From the $1^{\text {st }}$ row to the $9^{\text {th }}$, the images are from caudate, claustrum, cortex, hippocampus CA1 (cornu ammonis), hippocampus CA3, hippocampus dentate gyrus, putamen, subiculum and thalamus. a1-a9) Original images overlapped with expert annotations marked by red points. b1-b9) Estimated probability map of neuron centroid. c1-c9) Individualization results obtained by applying the proposed network. Centroids marked by white points are postprocessed based on b1-b9). Red contours are obtained by applying competitive region growing method You et al. (2019). d1-d9) Individualization results obtained based on You et al. (2019). e1-e9) Individualization results obtained by applying U-net (Ronneberger et al., 2015). f1-f9) Individualization results obtained by applying U-net++ (Zhou et al., 2020). g1g9) Individualization results obtained by applying FCRN (Xie et al., 2016). h1-h9) Individualization results obtained based on You et al. (2020). 
Figure 7 b1-b9. Pixels with greater intensities are more probable to be the neuron centroids. After post-processing and competitive region growing, neuron individualization results obtained by the proposed network are shown in Figure 7 c1-c9. Figure 7 d1-d9, e1-e9, f1-f9, g1-g9 and h1-h9 display neuron individualization results obtained by using unsupervised method proposed by You et al. (2019), deep learning methods of U-net (Ronneberger et al., 2015), Unet++ (Zhou et al., 2020), FCRN (Xie et al., 2016) and multiscale CNN (You et al., 2020), respectively. Thereinto, You's method (2019) and U-net++ are not good at individualizing elongated neuron (Figure $7 \mathrm{~d} 2 \& \mathrm{f} 2$ ). You's method (2019) leads easily over-individualization when neuron expression does not obey hypothesis based on Figure $7 \mathrm{~d} 2$, d4, d7 and d9. U-net and multiscale CNN often lead to neuron missed detection (Figure 7 e3, e 4 \& h4). The phenomenon of neuron under-individualization often presents in the situation similar to Figure 7 e3, e5-e7 and f3, f5-f9. Moreover, U-net and U-net++ cannot well detect and individualize highly aggregated neurons based on Figure 7 e6 and f6. FCRN introduces under-individualization in Figure $7 \mathrm{~g} 1$ and over-individualization in Figure $7 \mathrm{~g} 3$ and g5. Missed detection and under-individualization presents in Figure 7 h4, h6 and h7 by using Multiscale CNN. Using the proposed network can usually get good performance, but there may be neuron miss detection in the highly aggregated region like Figure $7 \mathrm{c} 6$. On the whole, in such a complex brain structure, the proposed network visually provides more accurate neuron detection and individualization performance.

\subsection{Comparison experiments of manual and automated neuron counting}

Neuron counting is important information in the field of biomedicine, so we count the number of neurons on the individualization dataset using unsupervised methods proposed by You et al. (2019) and deep learning methods of the proposed network, U-net (Ronneberger et al., 2015), U-net++ (Zhou et al., 2020), FCRN (Xie et al., 2016) and multiscale CNN (You et al., 2020). They respectively detect 111542, 106076, 85532, 94842, 101819, and 87320 neurons while a total of 111971 neurons were marked by expert (Table 1). The total number of neurons detected by the unsupervised method (You et al., 2019) and the proposed method are respectively the first and second method close to the expert's counting.

Table 1. Neuron counting and relative error by using different methods.

\begin{tabular}{lccccccc}
\hline & $\begin{array}{c}\text { Expert } \\
\text { counting }\end{array}$ & $\begin{array}{c}\text { Proposed } \\
\text { method }\end{array}$ & $\begin{array}{c}\text { Unsupervised } \\
\text { method } \\
\text { (You et al., } \\
\text { 2019) }\end{array}$ & $\begin{array}{c}\text { U-net } \\
\text { (Ronneberger } \\
\text { et al., 2015) }\end{array}$ & $\begin{array}{c}\text { U-net++ } \\
\text { (Zhou et al., } \\
\text { 2020) }\end{array}$ & $\begin{array}{c}\text { FCRN } \\
\text { (Xie et al., } \\
2016)\end{array}$ & $\begin{array}{c}\text { Multiscale } \\
\text { CNN } \\
\text { (You et al., } \\
\text { 2020) }\end{array}$ \\
\hline Number of neurons & 111971 & 106076 & 111542 & 85532 & 94842 & 101819 & 87320 \\
Relative error $(\%)$ & $/$ & 5.26 & 0.38 & 23.61 & 15.30 & 9.07 & 22.02 \\
\hline
\end{tabular}

\subsection{Comparison of automated neuron individualization}

Besides the number of neurons, the quality of neuron individualization is important. Therefore, an evaluation by using different methods based on F-score (equation $R=\frac{N_{t}}{N_{e}} ; \quad P=\frac{N_{t}}{N_{a}} ; \quad F=2 \frac{R \times P}{R+P}$

(4)) was performed.

Figure 8 shows the F-scores obtained by different automated methods applied on the individualization dataset. We observe that in the regions of caudate and subiculum, all the automated methods have good performance, almost all the F-scores are greater than 0.8. In the regions of claustrum, putamen, thalamus and cortex, the performance of different automated methods differs because both light- and dark- stained neurons, size-varying and touching neurons and neurons of different shapes are present which increase the difficulty in correctly detecting neurons. As for the challenging heterogeneous hippocampus region in neuroscience, the performance of all the methods are decreased. However, in general, we can still find that the proposed network can provide excellent individualization performance for any anatomical region. 


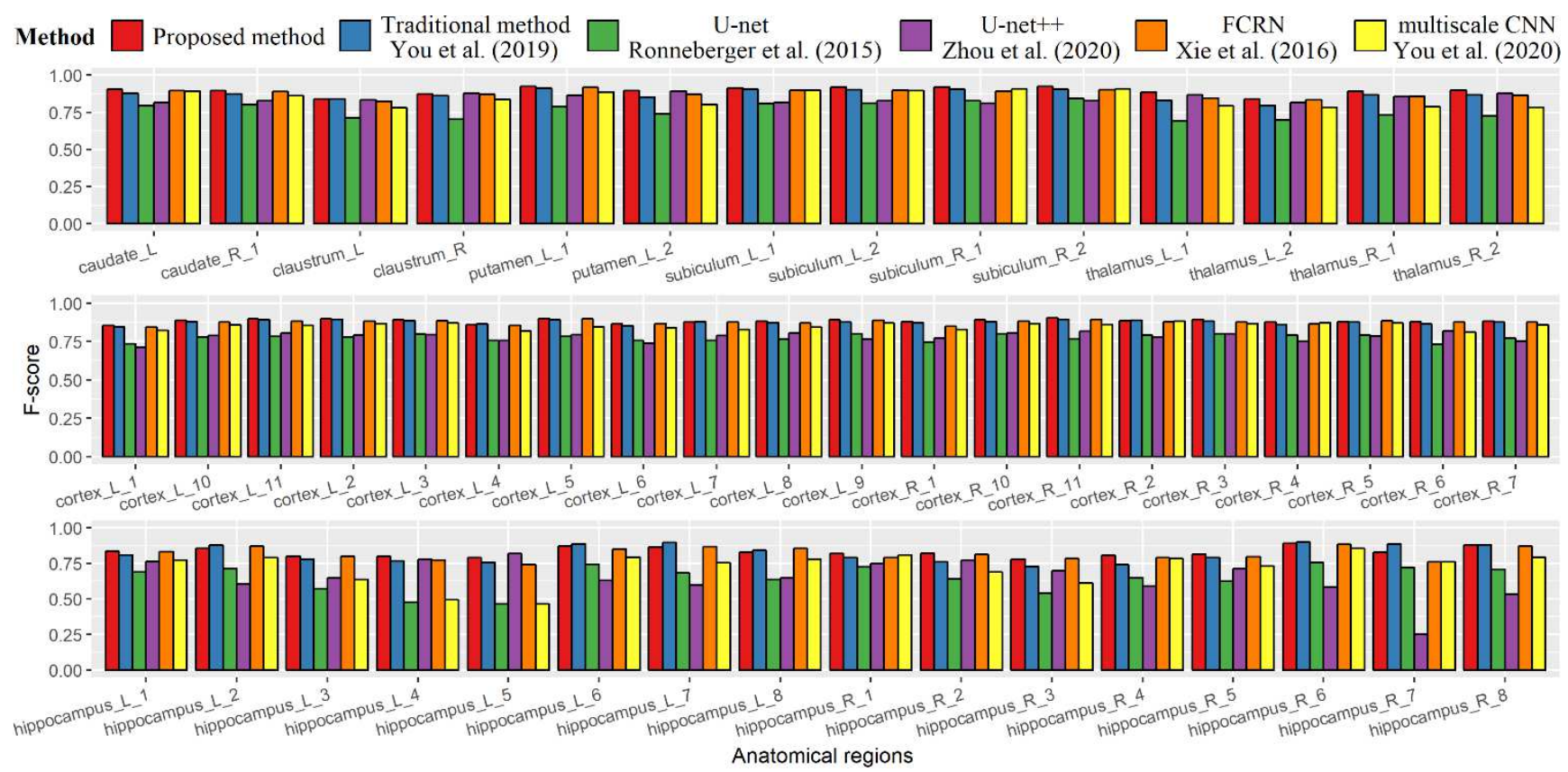

Figure 8. F-score calculated using the proposed method on 50 images of the individualization dataset. L (respectively, R) means that this image is extracted from the left (respectively, right) side of the section.

The average F-score for each anatomical region is presented in Table 2. We observe that the average F-score of the proposed method is the highest in any anatomical region. Except for the complex heterogenous hippocampus region, the average F-score values are all greater than 0.8577. Particularly, in the regions of caudate, putamen and subiculum, the average F-score values are greater than 0.9. The F-scores of hippocampus region varying from 0.7793 to 0.8896 are lower than other anatomical regions, which is due to the fact that hippocampus is a very complex heterogenous region. It contains different kinds and distributions of neurons which do not follow general NeuN-stained pattern. Like in Figure 7 a4 \& a5, different staining of these kinds of neurons (lighter staining in neuron center position) may be due to unsuitable staining marker (Mullen et al., 1992) corresponding to different levels of antigen expression. Nevertheless, the proposed network successfully individualized most neurons in hippocampus, and the calculated average F-score of 0.8287 improved respectively $1.5 \%, 28.4 \%, 27.9 \%, 1.6 \%$ and $15.3 \%$ compared to the unsupervised method proposed by You et al. (2019), deep learning techniques of U-net (Ronneberger et al., 2015), U-net++ (Zhou et al., 2020), FCRN

Table 2. Average F-score (average value \pm standard deviation) computed using different automated methods on the individualization dataset.

\begin{tabular}{ccccccc}
\hline $\begin{array}{c}\text { Anatomical } \\
\text { regions }\end{array}$ & $\begin{array}{c}\text { Proposed } \\
\text { method }\end{array}$ & $\begin{array}{c}\text { Unsupervised } \\
\text { method } \\
\text { (You et al., } \\
2019)\end{array}$ & $\begin{array}{c}\text { U-net } \\
\text { (Ronneberger } \\
\text { et al., 2015) }\end{array}$ & $\begin{array}{c}\text { U-net++ } \\
\text { (Zhou et al., } \\
\text { 2020) }\end{array}$ & $\begin{array}{c}\text { FCRN } \\
\text { (Xie et al., } \\
\text { 2016) }\end{array}$ & $\begin{array}{c}\text { Multiscale } \\
\text { CNN } \\
\text { (You et al., } \\
\text { 2020) }\end{array}$ \\
\hline Caudate & $\mathbf{0 . 9 0 1 4} \pm 0.0068$ & $0.8745 \pm 0.0029$ & $0.7997 \pm 0.0036$ & $0.8198 \pm 0.0082$ & $0.8921 \pm 0.0050$ & $0.8767 \pm 0.0217$ \\
Claustrum & $\mathbf{0 . 8 5 7 7} \pm 0.0238$ & $0.8508 \pm 0.0145$ & $0.7067 \pm 0.0069$ & $0.8545 \pm 0.0297$ & $0.8479 \pm 0.0327$ & $0.8073 \pm 0.0393$ \\
Cortex & $\mathbf{0 . 8 8 4 0 \pm 0 . 0 1 3 1}$ & $0.8767 \pm 0.0135$ & $0.7743 \pm 0.0220$ & $0.7805 \pm 0.0280$ & $0.8757 \pm 0.0139$ & $0.8519 \pm 0.0208$ \\
Hippocampus & $\mathbf{0 . 8 2 8 7} \pm 0.0331$ & $0.8165 \pm 0.0616$ & $0.6454 \pm 0.0906$ & $0.6477 \pm 0.1350$ & $0.8156 \pm 0.0444$ & $0.7187 \pm 0.1123$ \\
Putamen & $\mathbf{0 . 9 0 9 4} \pm 0.0215$ & $0.8811 \pm 0.0417$ & $0.7648 \pm 0.0356$ & $0.8783 \pm 0.0209$ & $0.8927 \pm 0.0332$ & $0.8430 \pm 0.0507$ \\
Subiculum & $\mathbf{0 . 9 1 9 6} \pm 0.0046$ & $0.9046 \pm 0.0015$ & $0.8221 \pm 0.0150$ & $0.8196 \pm 0.0081$ & $0.8976 \pm 0.0032$ & $0.9023 \pm 0.0050$ \\
Thalamus & $\mathbf{0 . 8 7 8 9} \pm 0.0265$ & $0.8408 \pm 0.0344$ & $0.7114 \pm 0.0184$ & $0.8527 \pm 0.0274$ & $0.8497 \pm 0.0145$ & $0.7869 \pm 0.0049$ \\
\hline Average & $\mathbf{0 . 8 6 9 4} \pm \mathbf{0 . 0 3 7 7}$ & $0.8559 \pm 0.0478$ & $0.7298 \pm 0.0830$ & $0.7554 \pm 0.1112$ & $0.8564 \pm 0.0411$ & $0.8070 \pm 0.0924$ \\
\hline
\end{tabular}


(Xie et al., 2016) and multiscale CNN (You et al., 2020)). In addition, the average F-score value of all the anatomical regions obtained by the proposed network is also the highest (respectively $1.6 \%, 19.1 \%, 15.1 \%, 1.5 \%$, and $7.7 \%$ improvement compared to reference methods). Combined with the smallest standard deviation, the proposed network provides the best and the most robust performance of neuron individualization for both simple and complex cases in macaque brain data.

\subsection{Individualization results with different number of images in the training set}

The influence of different number of images in the training set to the individualization performance of automated methods is studied. The average F-score of all the images of the individualization dataset is presented in Table 3 . The number of training set is respectively $152,304,456$ and 608 , i.e. $2 \%, 4 \%, 6 \%$ and $8 \%$ of the dataset size. Since You et al. (2019) proposed unsupervised method to individualize neurons, their F-score has no correlation with the number of training set. For the remaining deep learning methods except multiscale CNN (You et al., 2020), the average F-scores are increased with the number of training set. The F-score of multiscale CNN increased first and then decreased, that is because this method is specially designed for the detection of highly aggregated neurons. It cannot correctly extract features of neurons of different sizes. The individualization dataset is composed of the major anatomical regions of macaque brain data which concerns not only highly aggregated neurons but also size-varying neurons. Therefore, it leads to a performance decrease when the number of training set increases. Overall, we observe that among all the methods in Table 3, the proposed network consistently provides the best (the largest average F-score) and the most robust (the smallest standard deviation) individualization performance.

Table 3. Average F-score (average value \pm standard deviation) computed on the individualization dataset using different automated methods with different numbers of training set.

\begin{tabular}{ccccccc}
\hline $\begin{array}{c}\text { Number of } \\
\text { images in the } \\
\text { training set (\%) }\end{array}$ & $\begin{array}{c}\text { Proposed } \\
\text { method }\end{array}$ & $\begin{array}{c}\text { Unsupervised } \\
\text { method } \\
\text { (You et al., } \\
\text { 2019) }\end{array}$ & $\begin{array}{c}\text { U-net } \\
\text { (Ronneberger } \\
\text { et al., 2015) }\end{array}$ & $\begin{array}{c}\text { U-net++ } \\
\text { (Zhou et al., } \\
2020)\end{array}$ & $\begin{array}{c}\text { FCRN } \\
\text { (Xie et al., } \\
2016)\end{array}$ & $\begin{array}{c}\text { Multiscale } \\
\text { CNN } \\
\text { (You et al., } \\
\text { 2020) }\end{array}$ \\
\hline $152(2 \%)$ & $\mathbf{0 . 8 6 9 4 \pm 0 . 0 3 7 7}$ & & $0.7298 \pm 0.0830$ & $0.7554 \pm 0.1112$ & $0.8564 \pm 0.0411$ & $0.8070 \pm 0.0924$ \\
$304(4 \%)$ & $\mathbf{0 . 8 6 9 9} \pm \mathbf{0 . 0 4 1 6}$ & & $0.7414 \pm 0.0750$ & $0.7692 \pm 0.1059$ & $0.8625 \pm 0.0405$ & $0.8367 \pm 0.0605$ \\
$456(6 \%)$ & $\mathbf{0 . 8 7 1 5} \pm \mathbf{0 . 0 3 9 3}$ & $0.8559 \pm 0.0478$ & $0.7507 \pm 0.0641$ & $0.7784 \pm 0.1038$ & $0.8628 \pm 0.0511$ & $0.7997 \pm 0.0657$ \\
$608(8 \%)$ & $\mathbf{0 . 8 7 6 3} \pm \mathbf{0 . 0 4 2 2}$ & & $0.7587 \pm 0.0639$ & $/$ & $0.8679 \pm 0.0463$ & $0.7332 \pm 0.0856$ \\
\hline average & $\mathbf{0 . 8 7 1 8} \pm \mathbf{0 . 0 0 3 1}$ & $0.8559 \pm 0.0478$ & $0.7452 \pm 0.0124$ & $0.7677 \pm 0.0116$ & $0.8624 \pm 0.0047$ & $0.7942 \pm 0.0437$ \\
\hline
\end{tabular}

\subsection{Representation of neuron features in anatomical region}

Based on "neuron vector" computed at neuron level, several types of cartography can be generated. The number of neurons in anatomical regions of the brain is a major focus of interest for biologists. Figure 9 b1-b8 show the cartographies of the number of neurons in 6 regions of interest (stereology dataset shown in Figure 9 a1-a8) reflecting the density of neurons. The pixel aggregation has been fixed at $512 \times 512$ pixels which produces a new resolution of $0.11264 \mathrm{~mm} /$ pixel in both horizontal and vertical directions. Visually, the regions from higher densities to lower densities are in order: dentate gyrus, cortex, subiculum, caudate, claustrum, and thalamus. In the cortex, especially the Figure 9 b1, we observe very dense bands of neurons that correspond to different known layers of the cerebral cortex (Kolb et al., 1995). Figure $9 \mathrm{c1-c8}$ show the cartographies of the average orientation in 6 regions of interest. It is interesting to note that in the cortex (Figure $9 \mathrm{c} 1$ ) we do not find the very thin and intense bands like Figure 9 a1 or b1, the orientation of the neurons is stable and varies continuously. The orientations of neurons inside and around the caudate are different (Figure $9 \mathrm{c} 2$ ). In the claustrum (Figure $9 \mathrm{c} 8$ ), neurons present obvious directionality, which is corresponding to Figure 7 a2. The orientation of neurons is much more variable in the thalamus (Figure 9 c3 \& c6) which is known to consist of many sub-neurons with different organizations (Dauguet et al., 2009). 


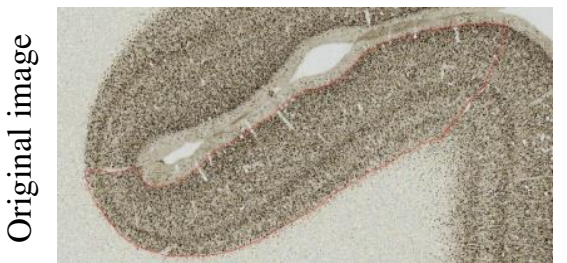

a1) S1_cortex

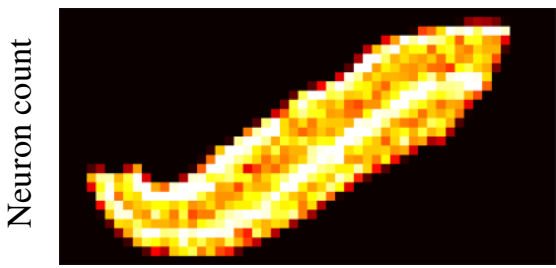

b1) $[0,67]$

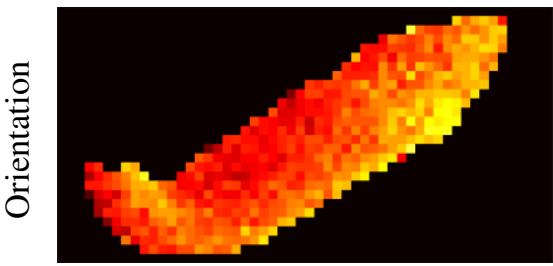

c1) $\left[-88.1^{\circ}, 5^{\circ} .7^{\circ}\right]$

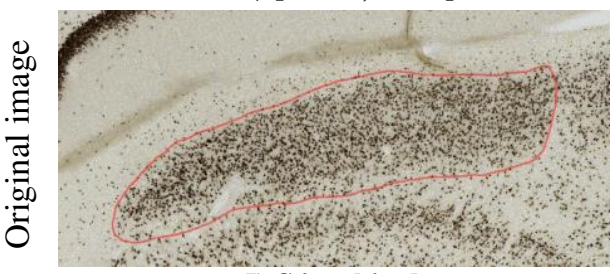

a5) S4_subiculum

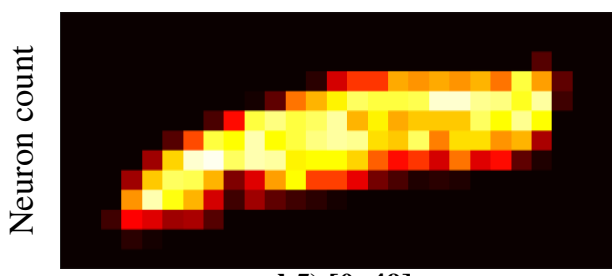

b5) $[0,49]$

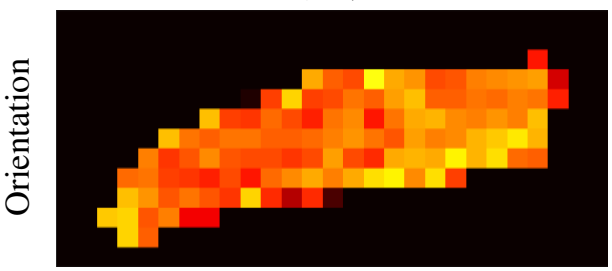

c5) $\left[-82.9^{\circ}, 47.5^{\circ}\right]$

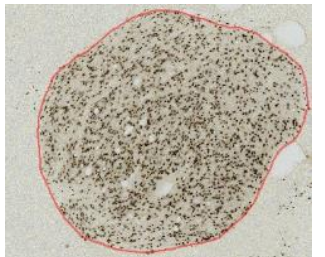

a2) S2_caudate

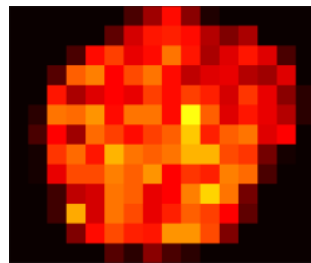

b2) $[0,38]$

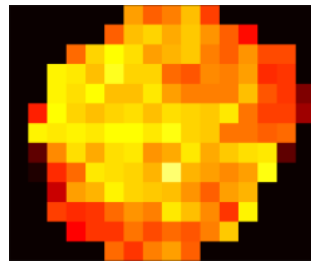

c2) $\left[-83.0^{\circ}, 63.3^{\circ}\right]$

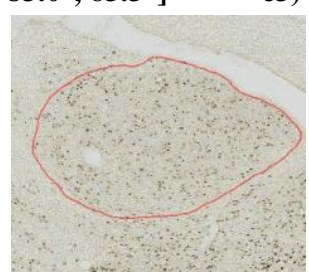

a6) S5 thalamus

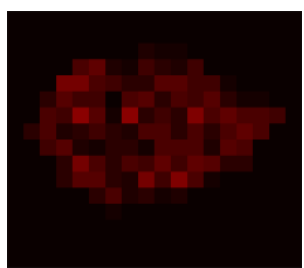

b6) $[0,10]$

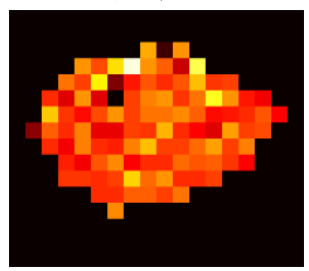

c6) $\left[-73.5^{\circ}, 8^{\circ 3.2^{\circ}}\right]$

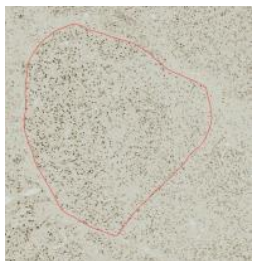

a3) S3 thalamus

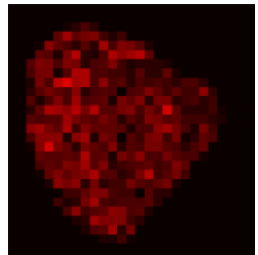

b3) $[0,16]$

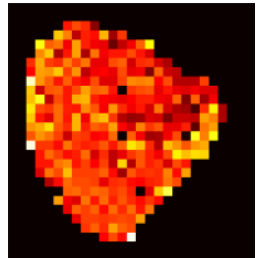

c3) $\left[-83.3^{\circ}, 86.8^{\circ}\right]$

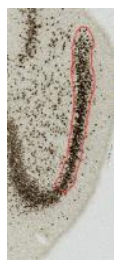

a7) S6_gyrus

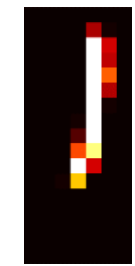

b7) $[0,76$

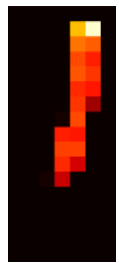

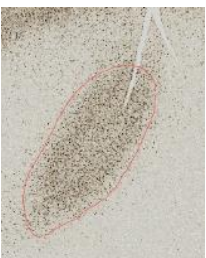

a4) S7_cortex

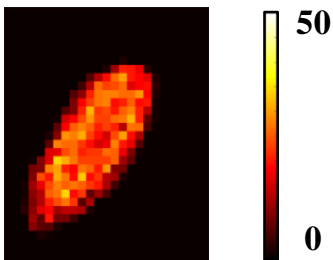

b4) $[0,34]$

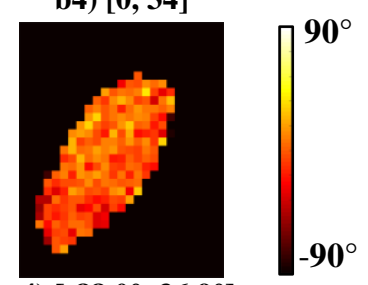

c4) $\left[-88.0^{\circ}, 36.9^{\circ}\right]$

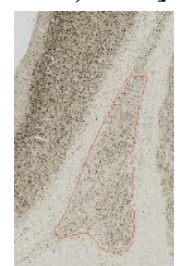

a8) S8_claustrum

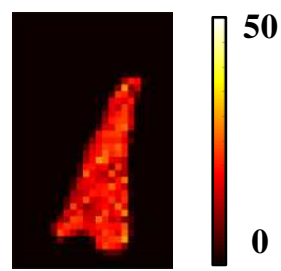

b8) $[0,30]$

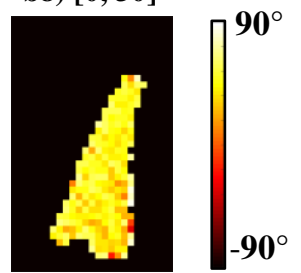

c7) $\left[-86.8^{\circ}, 81.3^{\circ}\right] \quad$ c8) $\left[-31.9^{\circ}, 85.1^{\circ}\right]$

Figure 9. Representation of cartography of two descriptors (the number of neurons and the average of orientation of neurons) in 6 anatomical regions of interest. The aggregation faction is 512 pixels in both horizontal and vertical directions. The resolution at mesoscopic level is $0.11264 \mathrm{~mm} /$ pixel. a1) S1_cortex. a2) S2_caudate. a3) S3_thalamus. a4) S7_cortex. a5) S4_subiculum. a6) S5_thalamus. a7) S6_dentate_gyrus. a8) S8_claustrum. b1-b8) are respectively the cartography of the number of neurons and c1-c8) are respectively the cartography of the average of orientation of neurons. The range of the value of descriptors represented by each pixel is listed at right bottom of the image.

\section{Discussion}

We proposed a novel scheme of multiscale fully convolutional regression neural network combined with competitive 
region growing technique to individualize size-varying and touching neurons in major anatomical regions of macaque brain using only point annotations for training. Thanks to the multiscale resolution achieved by parallel multiple receptive fields and different network depths, our proposed network succeeds in detecting the centroids of size-varying and touching neurons. Competitive region growing applied on these centroids achieves neuron individualization which will help study the morphology and distribution of neurons in the entire brain.

The proposed method provides satisfying individualization performance both qualitatively and quantitatively. Figure 7 shows examples of excellent individualization results obtained by the proposed method in different anatomical regions. Based on Figure 8, the proposed method can best individualize neurons in most images compared to the reference methods. However, in the image of hippocampus_L_2, hippocampus_L_6, hippocampus_L_7, hippocampus_L_8, hippocampus_R_6, hippocampus_R_7 and hippocampus_R_8, neurons individualized by the proposed method are not as good as unsupervised method proposed by You et al. (2019). That is because dentate gyrus, a special anatomical region containing thousands of small-sized touching neurons, exist in these images. The unsupervised method proposed by You et al. (2019) concluded that the size of the neurons in dentate gyrus is stable, so they fixed a parameter specially for neuron individualization of this region resulting in better individualization results than those obtained by deep learning methods which use the unique architecture for all anatomical regions. Nevertheless, the F-scores of the proposed method are still competitive in regard of those calculated by using the unsupervised method. They are respectively 0.8548 vs. 0.8748 for hippocampus_L_2, 0.8680 vs. 0.8856 for hippocampus_L_6, 0.8618 vs. 0.8954 for hippocampus_L_7, 0.8271 vs. 0.8404 for hippocampus_L_8, 0.8896 vs. 0.8976 for hippocampus_R_6, 0.8259 vs. 0.8845 for hippocampus_R_7 and 0.8784 vs. 0.8782 for hippocampus_R_8. The average F-score of 0.8287 in hippocampus region calculated by the proposed method reaches a $1.5 \%$ performance improvement compared to the unsupervised method, demonstrating better individualization results in the other hippocampus subregions (CA1-CA4) though containing different kinds and distributions of neurons. In any case, the hippocampus has always been the most challenging anatomical region in neuroscience. It is still worth studying this region.

According to Table 2, the average F-scores of the proposed method is the highest in any anatomical region. Such satisfying results must be attributed to the design of parallel multiple receptive fields and appropriate network depth. The proposed network implementing a total of 38 receptive fields with the size from $1 \times 1$ to $284 \times 284$ pixels successfully extracts the features of different sizes of neurons $(30 \mu \mathrm{m}$ in diameter in maximum, i.e. 137 pixels). The extraction of richer features of neurons succeeds in individualizing single individual, size-varying and touching neurons.

Table 3 reflects the influence of different numbers of training set to the individualization performance of automated methods. The proposed method always presents the best and the most robust individualization performances. As the number of training set doubles, neuron individualization performance increases by $0.55 \%$ at most. Therefore, we can use small samples to train the network model to perform satisfying neuron individualization of the entire brain and/or perform data augmentation to improve the individualization performance. This can alleviate the marking work of experts.

Light-sheet microscopy allowing for very high spatial resolution down to the cellular level has been a good alternative to image large samples. However, it also generates very heavy data. The cartography makes it possible to synthesize the relevant information, to transform this massive qualitative data into quantitative data that is more informative and easier to analyze. Figure 9 provides an initial impression of the cartography of the number and the average orientation of neurons of different anatomical regions. The combination of these different information can, for example, be used to segment the cortical layers which constitute a very active field of research in neuroscience. In addition, it provides possibility of comparison with other types of imaging modalities such as Magnetic Resonance Imaging.

Neuron individualization is still an essential and challenging research in neuroscience. Various unsupervised methods and deep learning methods have demonstrated their efficiency over the past years. However, most of them only focused on special anatomical regions where both of the neuron expression and distribution are simple. This is because that the $2 \mathrm{D}$ images are obtained through a single scanning focus plan which makes it difficult to individualize touching neurons, especially touching neurons of different sizes. In order to get a better and more general individualization method, it may be better to work in 3D. But there will be new problems, that is, 3D imaging modalities are limited in the field of view of light-sheet imaging and the performance of most computers cannot afford to handle such large 3D volume data (Renier et al., 2014).

The proposed network can help address major biological challenges, such as improving our understanding of brain 
development or aging, deciphering pathology mechanisms, or evaluating novel therapies in neurodegenerative diseases.

\section{Conclusion}

This paper presents a new image processing protocol to individualize size-varying and touching neurons in major anatomical regions in macaque brain based on deep learning approach. The performance of neuron detection and individualization obtained by the proposed protocol outperforms state-of-the-art methods in both qualitative and quantitative assessment. Since the automated neuron individualization in macaque brain is challenging, an in-depth comparison of strengths and complementarities between stereology and image processing methods should be carefully addressed in the future. In terms of perspectives, this work will be exploited to perform neuron individualization at large scale in entire brain section in order to analyze neurons morphology and distribution. It will provide new tools to explore brain development, aging and neurodegenerative diseases.

\section{Data availability statement}

The datasets applied in this paper come from MIRCen CEA France (Molecular Imaging Research Center, The French Alternative Energies and Atomic Energy Commission). The data will be available later in the form of an article and an access because it corresponds to a large amount of information.

\section{Ethics statement}

The animal study was reviewed and approved by the Comité d'éthique agréé par le MESR dont relève l'EU: CETEA DSV-Comité n॰44.

\section{Conflicts of interest}

The authors declare that they have no conflict of interest.

\section{Funding}

This work was supported by Natural Science Foundation of Shaanxi Province [2020JQ-652]; Natural Science Foundation of Shaanxi Provincial Department of Education [20JK0795]; Fund of Doctoral Start-up of Xi'an University of Technology [112/256081811]; French national funds (PIA2' program) under contract No. P112331-3422142 (3D NeuroSecure project).

\section{References}

Adams, R., Bischof, L., 1994. Seeded region growing. IEEE Trans. Pattern Anal. Mach. Intell. 16, $641-647$. https://doi.org/10.1109/34.295913

Al-Kofahi, Y., Lassoued, W., Lee, W., Roysam, B., 2010. Improved Automatic Detection and Segmentation of Cell Nuclei in Histopathology Images. IEEE Trans. Biomed. Eng. 57, 841-852. https://doi.org/10.1109/TBME.2009.2035102

Andersen, P., Morris, R., Amaral, D., Bliss, T., O’Keefe, J., 2016. The Hippocampus Book. Oxford University Press.

Andrey, P., Kiêu, K., Kress, C., Lehmann, G., Tirichine, L., Liu, Z., Biot, E., Adenot, P.-G., Hue-Beauvais, C., HoubaHérin, N., Duranthon, V., Devinoy, E., Beaujean, N., Gaudin, V., Maurin, Y., Debey, P., 2010. Statistical Analysis of 3D Images Detects Regular Spatial Distributions of Centromeres and Chromocenters in Animal and Plant Nuclei. PLOS Comput. Biol. 6, e1000853. https://doi.org/10.1371/journal.pcbi.1000853

Bai, X., Sun, C., Zhou, F., 2008. Touching Cells Splitting by Using Concave Points and Ellipse Fitting, in: Digital Image Computing: Techniques and Applications (DICTA), 2008. Presented at the Digital Image Computing: Techniques and Applications (DICTA), 2008, pp. 271-278. https://doi.org/10.1109/DICTA.2008.11

Brabandere, B.D., Neven, D., Gool, L.V., 2017. Semantic Instance Segmentation for Autonomous Driving, in: 2017 IEEE Conference on Computer Vision and Pattern Recognition Workshops (CVPRW). Presented at the 2017 IEEE Conference on Computer Vision and Pattern Recognition Workshops (CVPRW), pp. 478-480. https://doi.org/10.1109/CVPRW.2017.66

Cheng, H.D., Jiang, X.H., Sun, Y., Wang, J.L., 2001. Color image segmentation: Advances and prospects. Pattern Recognit. 34, 2259-2281. 
Cousty, J., Bertrand, G., Najman, L., Couprie, M., 2009. Watershed Cuts: Minimum Spanning Forests and the Drop of Water Principle. IEEE Trans. Pattern Anal. Mach. Intell. 31, 1362-1374. https://doi.org/10.1109/TPAMI.2008.173

Daněk, O., Matula, P., Ortiz-de-Solórzano, C., Muñoz-Barrutia, A., Maška, M., Kozubek, M., 2009. Segmentation of Touching Cell Nuclei Using a Two-Stage Graph Cut Model, in: Image Analysis, Lecture Notes in Computer Science. Presented at the Scandinavian Conference on Image Analysis, Springer, Berlin, Heidelberg, pp. 410-419. https://doi.org/10.1007/978-3-642-02230-2_42

Dauguet, J., Condé, F., Hantraye, P., Frouin, V., Delzescaux, T., 2009. Generation of a 3D atlas of the nuclear division of the thalamus based on histological sections of primate: Intra- and intersubject atlas-to-MRI warping. IRBM, NUMÉRO SPÉCIAL TECHNOLOGIES POUR L'AUTONOMIE 30, 281-291. https://doi.org/10.1016/j.irbm.2009.10.004

Falk, T., Mai, D., Bensch, R., Çiçek, Ö., Abdulkadir, A., Marrakchi, Y., Böhm, A., Deubner, J., Jäckel, Z., Seiwald, K., Dovzhenko, A., Tietz, O., Dal Bosco, C., Walsh, S., Saltukoglu, D., Tay, T.L., Prinz, M., Palme, K., Simons, M., Diester, I., Brox, T., Ronneberger, O., 2019. U-Net: deep learning for cell counting, detection, and morphometry. Nat. Methods 16, 67-70. https://doi.org/10.1038/s41592-018-0261-2

Gundersen, H.J.G., 1986. Stereology of Arbitrarily Shaped Particles: Unbiased Estimation of Number and Sizes, in: Proceedings of the First International Symposium for Science on Form. Presented at the Proceedings of the First International Symposium for Science on Form.

He, K., Zhang, X., Ren, S., Sun, J., 2016. Deep Residual Learning for Image Recognition, in: 2016 IEEE Conference on Computer Vision and Pattern Recognition (CVPR). Presented at the 2016 IEEE Conference on Computer Vision and Pattern Recognition (CVPR), pp. 770-778. https://doi.org/10.1109/CVPR.2016.90

He, Y., Gong, H., Xiong, B., Xu, X., Li, A., Jiang, T., Sun, Q., Wang, S., Luo, Q., Chen, S., 2015. iCut: an Integrative Cut Algorithm Enables Accurate Segmentation of Touching Cells. Sci. Rep. 5. https://doi.org/10.1038/srep12089

Huang, Y., Zhu, H., Wang, P., Dong, D., 2019. Segmentation of Overlapping Cervical Smear Cells Based on U-Net and Improved Level Set, in: 2019 IEEE International Conference on Systems, Man and Cybernetics (SMC). Presented at the 2019 IEEE International Conference on Systems, Man and Cybernetics (SMC), pp. 3031-3035. https://doi.org/10.1109/SMC.2019.8914625

Jelsing, J., Nielsen, R., Olsen, A.K., Grand, N., Hemmingsen, R., Pakkenberg, B., 2006. The postnatal development of neocortical neurons and glial cells in the Göttingen minipig and the domestic pig brain. J. Exp. Biol. 209, 14541462. https://doi.org/10.1242/jeb.02141

Kainz, P., Urschler, M., Schulter, S., Wohlhart, P., Lepetit, V., 2015. You Should Use Regression to Detect Cells, in: Medical Image Computing and Computer-Assisted Intervention - MICCAI 2015, Lecture Notes in Computer Science. Presented at the International Conference on Medical Image Computing and Computer-Assisted Intervention, Springer, Cham, pp. 276-283. https://doi.org/10.1007/978-3-319-24574-4_33

Karlsen, A.S., Pakkenberg, B., 2011. Total numbers of neurons and glial cells in cortex and basal ganglia of aged brains with Down syndrome--a stereological study. Cereb. Cortex N. Y. N 1991 21, 2519-2524. https://doi.org/10.1093/cercor/bhr033

Kolb, H., Fernandez, E., Nelson, R. (Eds.), 1995. Webvision: The Organization of the Retina and Visual System. University of Utah Health Sciences Center, Salt Lake City (UT).

Kothari, S., Chaudry, Q., Wang, M.D., 2009. Automated cell counting and cluster segmentation using concavity detection and ellipse fitting techniques, in: IEEE International Symposium on Biomedical Imaging: From Nano to Macro, 2009. ISBI '09. Presented at the IEEE International Symposium on Biomedical Imaging: From Nano to Macro, 2009. ISBI '09, pp. 795-798. https://doi.org/10.1109/ISBI.2009.5193169

Larsen, C.C., Bonde Larsen, K., Bogdanovic, N., Laursen, H., Graem, N., Samuelsen, G.B., Pakkenberg, B., 2006. Total number of cells in the human newborn telencephalic wall. Neuroscience 139, 999-1003. https://doi.org/10.1016/j.neuroscience.2006.01.005

Li, C., Wang, X., Liu, W., Latecki, L.J., Wang, B., Huang, J., 2019. Weakly supervised mitosis detection in breast histopathology images using concentric loss. Med. Image Anal. 53, 165-178. https://doi.org/10.1016/j.media.2019.01.013

Lin, T.-Y., Dollár, P., Girshick, R., He, K., Hariharan, B., Belongie, S., 2017. Feature Pyramid Networks for Object Detection, in: 2017 IEEE Conference on Computer Vision and Pattern Recognition (CVPR). Presented at the 2017 IEEE Conference on Computer Vision and Pattern Recognition (CVPR), pp. 936-944. https://doi.org/10.1109/CVPR.2017.106 
Long, J., Shelhamer, E., Darrell, T., 2015. Fully convolutional networks for semantic segmentation, in: 2015 IEEE Conference on Computer Vision and Pattern Recognition (CVPR). Presented at the 2015 IEEE Conference on Computer Vision and Pattern Recognition (CVPR), pp. 3431-3440. https://doi.org/10.1109/CVPR.2015.7298965

Lou, X., Koethe, U., Wittbrodt, J., Hamprecht, F.A., 2012. Learning to segment dense cell nuclei with shape prior, in: 2012 IEEE Conference on Computer Vision and Pattern Recognition. Presented at the 2012 IEEE Conference on Computer Vision and Pattern Recognition, pp. 1012-1018. https://doi.org/10.1109/CVPR.2012.6247778

Ma, H., Beiter, R., Gaultier, A., Acton, S.T., Lin, Z., 2018. OSLO: Automatic Cell Counting and Segmentation for Oligodendrocyte Progenitor Cells, in: 2018 25th IEEE International Conference on Image Processing (ICIP). Presented at the 2018 25th IEEE International Conference on Image Processing (ICIP), pp. 2431-2435. https://doi.org/10.1109/ICIP.2018.8451410

Mullen, R.J., Buck, C.R., Smith, A.M., 1992. NeuN, a neuronal specific nuclear protein in vertebrates. Dev. Camb. Engl. 116, 201-211.

Naylor, P., Laé, M., Reyal, F., Walter, T., 2019. Segmentation of Nuclei in Histopathology Images by Deep Regression of the Distance Map. IEEE Trans. Med. Imaging 38, 448-459. https://doi.org/10.1109/TMI.2018.2865709

Nedzved, A., Ablameyko, S., Pitas, I., 2000. Morphological segmentation of histology cell images, in: 15th International Conference on Pattern Recognition. pp. 500-503. https://doi.org/10.1109/ICPR.2000.905385

Pakkenberg, B., Gundersen, H.J., 1997. Neocortical neuron number in humans: effect of sex and age. J. Comp. Neurol. 384, 312-320.

Passingham, R., 2009. How good is the macaque monkey model of the human brain? Curr. Opin. Neurobiol. 19, 6-11. https://doi.org/10.1016/j.conb.2009.01.002

Pelvig, D.P., Pakkenberg, H., Stark, A.K., Pakkenberg, B., 2008. Neocortical glial cell numbers in human brains. Neurobiol. Aging 29, 1754-1762. https://doi.org/10.1016/j.neurobiolaging.2007.04.013

Qi, J., 2014. Dense nuclei segmentation based on graph cut and convexity-concavity analysis. J. Microsc. 253, 42-53. https://doi.org/10.1111/jmi.12096

Rad, R.M., Saeedi, P., Au, J., Havelock, J., 2018. Multi-Resolutional Ensemble of Stacked Dilated U-Net for Inner Cell Mass Segmentation in Human Embryonic Images, in: 2018 25th IEEE International Conference on Image Processing (ICIP). Presented at the 2018 25th IEEE International Conference on Image Processing (ICIP), pp. 3518-3522. https://doi.org/10.1109/ICIP.2018.8451750

Raza, S.E.A., AbdulJabbar, K., Jamal-Hanjani, M., Veeriah, S., Quesne, J.L., Swanton, C., Yuan, Y., 2019. Deconvolving Convolutional Neural Network for Cell Detection, in: 2019 IEEE 16th International Symposium on Biomedical Imaging (ISBI 2019). Presented at the 2019 IEEE 16th International Symposium on Biomedical Imaging (ISBI 2019), pp. 891-894. https://doi.org/10.1109/ISBI.2019.8759333

Renier, N., Wu, Z., Simon, D.J., Yang, J., Ariel, P., Tessier-Lavigne, M., 2014. iDISCO: a simple, rapid method to immunolabel large tissue samples for volume imaging. Cell 159, 896-910. https://doi.org/10.1016/j.cell.2014.10.010

Riccio, D., Brancati, N., Frucci, M., Gragnaniello, D., 2019. A New Unsupervised Approach for Segmenting and Counting Cells in High-Throughput Microscopy Image Sets. IEEE J. Biomed. Health Inform. 23, 437-448. https://doi.org/10.1109/JBHI.2018.2817485

Ronneberger, O., Fischer, P., Brox, T., 2015. U-Net: Convolutional Networks for Biomedical Image Segmentation, in: Navab, N., Hornegger, J., Wells, W.M., Frangi, A.F. (Eds.), Medical Image Computing and Computer-Assisted Intervention - MICCAI 2015. Springer International Publishing, pp. 234-241.

Shu, J., Fu, H., Qiu, G., Kaye, P., Ilyas, M., 2013. Segmenting overlapping cell nuclei in digital histopathology images. Conf. Proc. Annu. Int. Conf. IEEE Eng. Med. Biol. Soc. IEEE Eng. Med. Biol. Soc. Annu. Conf. 2013, 54455448. https://doi.org/10.1109/EMBC.2013.6610781

Sofiiuk, K., Barinova, O., Konushin, A., 2019. AdaptIS: Adaptive Instance Selection Network, in: 2019 IEEE International Conference on Computer Vision (ICCV). Presented at the 2019 IEEE International Conference on Computer Vision (ICCV), pp. 7355-7363.

Thu, D.C., Oorschot, D.E., Tippett, L.J., Nana, A.L., Hogg, V.M., Synek, B.J., Luthicarter, R., Waldvogel, H.J., Faull, R.L., 2010. Cell loss in the motor and cingulate cortex correlates with symptomatology in Huntington's disease. Brain J. Neurol. 133, 1094-110.

Ulman, V., Maška, M., Magnusson, K.E.G., Ronneberger, O., Haubold, C., Harder, N., Matula, Pavel, Matula, Petr, Svoboda, D., Radojevic, M., Smal, I., Rohr, K., Jaldén, J., Blau, H.M., Dzyubachyk, O., Lelieveldt, B., Xiao, P., Li, Y., Cho, S.-Y., Dufour, A.C., Olivo-Marin, J.-C., Reyes-Aldasoro, C.C., Solis-Lemus, J.A., Bensch, R., Brox, T., Stegmaier, J., Mikut, R., Wolf, S., Hamprecht, F.A., Esteves, T., Quelhas, P., Demirel, Ö., Malmström, L., Jug, F., Tomancak, P., Meijering, E., Muñoz-Barrutia, A., Kozubek, M., Ortiz-de-Solorzano, C., 2017. An 
objective comparison of cell-tracking algorithms. Nat. Methods 14, 1141-1152.

https://doi.org/10.1038/nmeth.4473

Vicar, T., Raudenska, M., Gumulec, J., Balvan, J., 2020. The Quantitative-Phase Dynamics of Apoptosis and Lytic Cell Death. Sci. Rep. 10. https://doi.org/10.1038/s41598-020-58474-w

Vuola, A.O., Akram, S.U., Kannala, J., 2019. Mask-RCNN and U-Net Ensembled for Nuclei Segmentation, in: 2019 IEEE 16th International Symposium on Biomedical Imaging (ISBI 2019). Presented at the 2019 IEEE 16th International Symposium on Biomedical Imaging (ISBI 2019), pp. 208-212. https://doi.org/10.1109/ISBI.2019.8759574

Waldvogel, H.J., Kim, E.H., Tippett, L.J., Vonsattel, J.P.G., Faull, R.L., 2015. The Neuropathology of Huntington's Disease. Curr. Top. Behav. Neurosci. 22, 33.

Walløe, S., Pakkenberg, B., Fabricius, K., 2014. Stereological estimation of total cell numbers in the human cerebral and cerebellar cortex. Front. Hum. Neurosci. 8, 508. https://doi.org/10.3389/fnhum.2014.00508

Wang, X., Kong, T., Shen, C., Jiang, Y., Li, L., 2020. SOLO: Segmenting Objects by Locations. ArXiv191204488 Cs.

West, M.J., Slomianka, L., Gundersen, H.J., 1991. Unbiased stereological estimation of the total number of neurons in the subdivisions of the rat hippocampus using the optical fractionator. Anat. Rec. 231, 482-497. https://doi.org/10.1002/ar.1092310411

Williams, R.W., Herrup, K., 1988. The control of neuron number. Annu. Rev. Neurosci. 11, 423-453. https://doi.org/10.1146/annurev.ne.11.030188.002231

Xie, W., Noble, J.A., Zisserman, A., 2016. Microscopy cell counting and detection with fully convolutional regression networks. Comput. Methods Biomech. Biomed. Eng. Imaging Vis. 6, 283-292.

Xue, Y., Ray, N., Hugh, J., Bigras, G., 2017. A novel framework to integrate convolutional neural network with compressed sensing for cell detection, in: 2017 IEEE International Conference on Image Processing (ICIP). Presented at the 2017 IEEE International Conference on Image Processing (ICIP), pp. 2319-2323. https://doi.org/10.1109/ICIP.2017.8296696

Yang, X., Li, H., Zhou, X., 2006. Nuclei Segmentation Using Marker-Controlled Watershed, Tracking Using Mean-Shift, and Kalman Filter in Time-Lapse Microscopy. IEEE Trans. Circuits Syst. Regul. Pap. 53, 2405-2414. https://doi.org/10.1109/TCSI.2006.884469

Yoo, I., Yoo, D., Paeng, K., 2019. PseudoEdgeNet: Nuclei Segmentation only with Point Annotations, in: Shen, D., Liu, T., Peters, T.M., Staib, L.H., Essert, C., Zhou, S., Yap, P.-T., Khan, A. (Eds.), Medical Image Computing and Computer Assisted Intervention - MICCAI 2019, Lecture Notes in Computer Science. Springer International Publishing, Cham, pp. 731-739. https://doi.org/10.1007/978-3-030-32239-7_81

You, Z., Balbastre, Y., Bouvier, C., Hérard, A.-S., Gipchtein, P., Hantraye, P., Jan, C., Souedet, N., Delzescaux, T., 2019. Automated Individualization of Size-Varying and Touching Neurons in Macaque Cerebral Microscopic Images. Front. Neuroanat. 13. https://doi.org/10.3389/fnana.2019.00098

You, Z., Jiang, M., Shi, Z., Shi, C., Du, S., Liang, J., Hérard, A.-S., Jan, C., Souedet, N., Delzescaux, T., 2020. Automated Detection Of Highly Aggregated Neurons In Microscopic Images Of Macaque Brain, in: 2020 IEEE International Conference on Image Processing (ICIP). Presented at the 2020 IEEE International Conference on Image Processing (ICIP), pp. 315-319. https://doi.org/10.1109/ICIP40778.2020.9190747

Zhang, C., Sun, C., Pham, T.D., 2013. Segmentation of clustered nuclei based on concave curve expansion. J. Microsc. 251, 57-67. https://doi.org/10.1111/jmi.12043

Zhang, J., Hu, Z., Han, G., He, X., 2016. Segmentation of overlapping cells in cervical smears based on spatial relationship and Overlapping Translucency Light Transmission Model. Pattern Recognit. 60, 286-295. https://doi.org/10.1016/j.patcog.2016.04.021

Zhou, Z., Siddiquee, M.M.R., Tajbakhsh, N., Liang, J., 2020. UNet++: Redesigning Skip Connections to Exploit Multiscale Features in Image Segmentation. IEEE Trans. Med. Imaging 39, 1856-1867. https://doi.org/10.1109/TMI.2019.2959609

Zucker, S.W., 1976. Region Growing: childhood and adolescence. Comput. Graph. Image Process. 5, 382-399. 


\section{Figures}

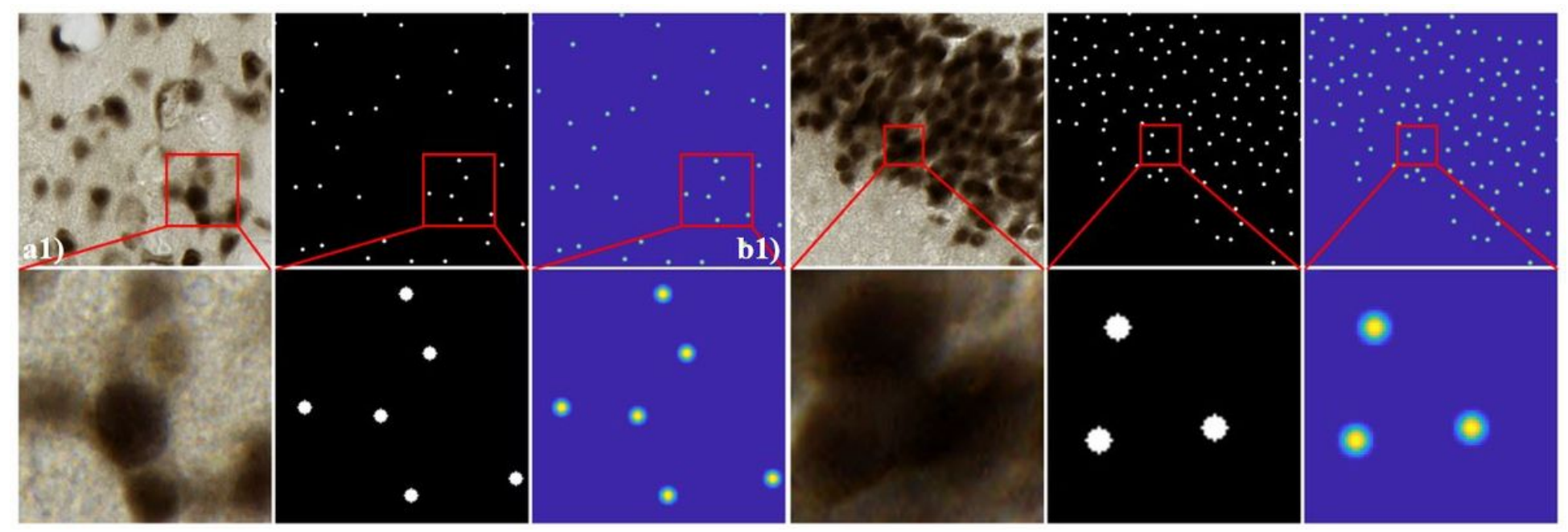

\section{Figure 1}

Example images of size-varying and touching neurons. a1 \& a2) Example original images of cortex and hippocampus dentate gyrus. b1 \& b2) Expert annotations (disks with a radius of 5 pixels in center location of neurons) on images of a1 \& a2). c1 \& c2) Preprocessing on expert annotations using a Gaussian filter ( $\sigma=3$ pixels).
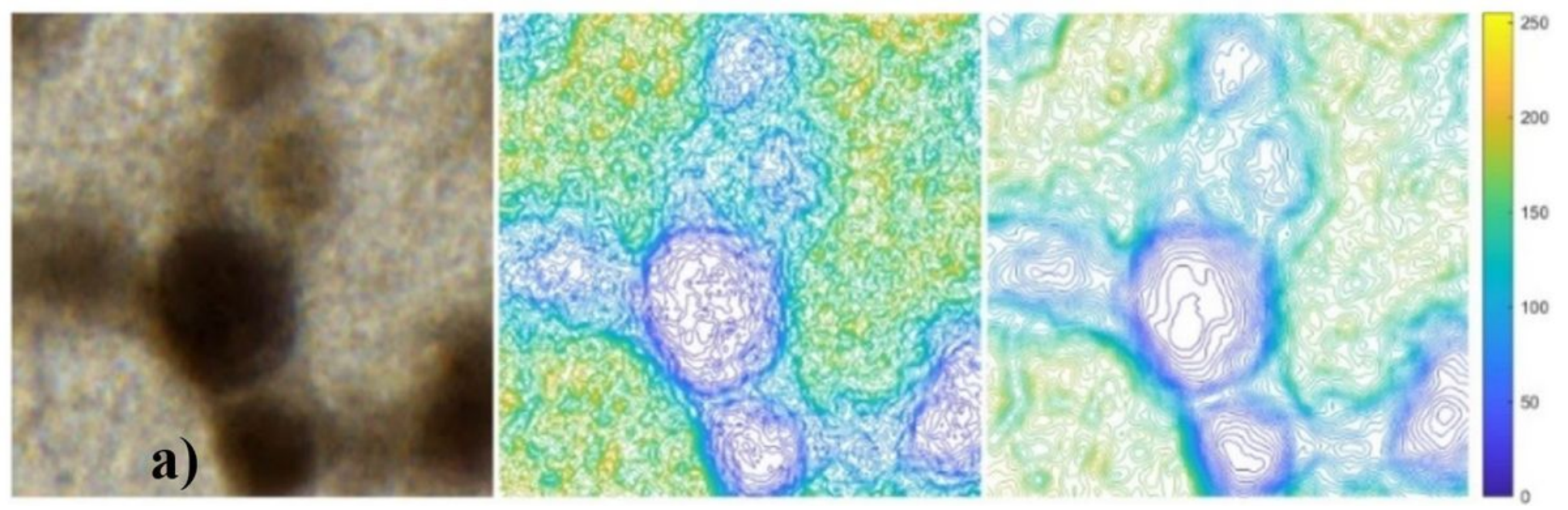

\section{Figure 2}

Characteristics of NeuN-stained neurons. a) Original color image in the cortex. b) Contour image based on pixel intensity of a). c) Denoising image of $b$ ) using a Gaussian filter ( $\sigma=3$ pixels). 


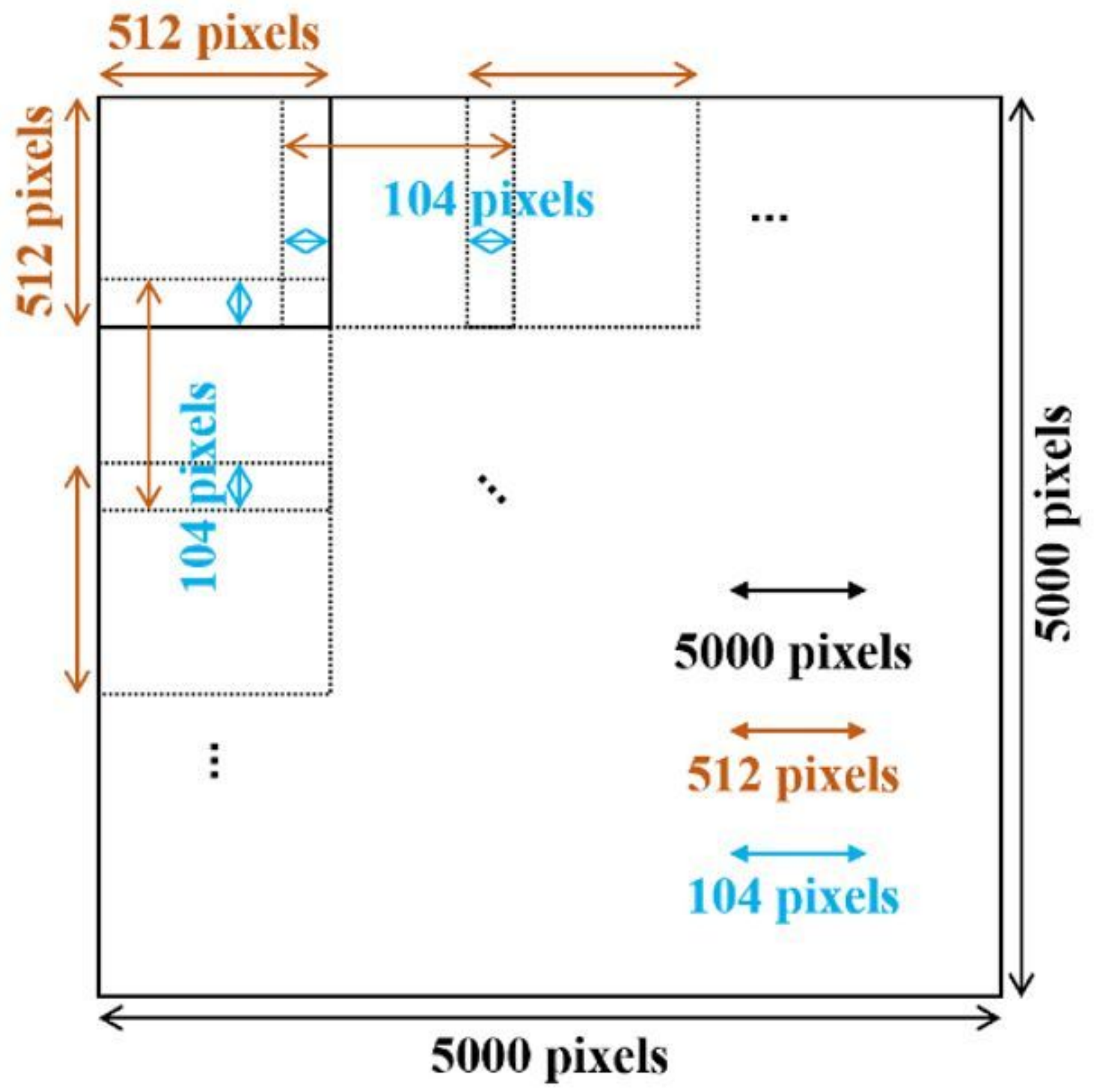

Figure 3

Image cropping. Each image of size $5000 \times 5000$ pixels from individualization dataset from You et al. (2019) was cropped to 144 small images of size $512 \times 512$ pixels adapted for deep learning. There are 104 pixels overlapped between each two adjacent images in both horizontal and vertical direction. 


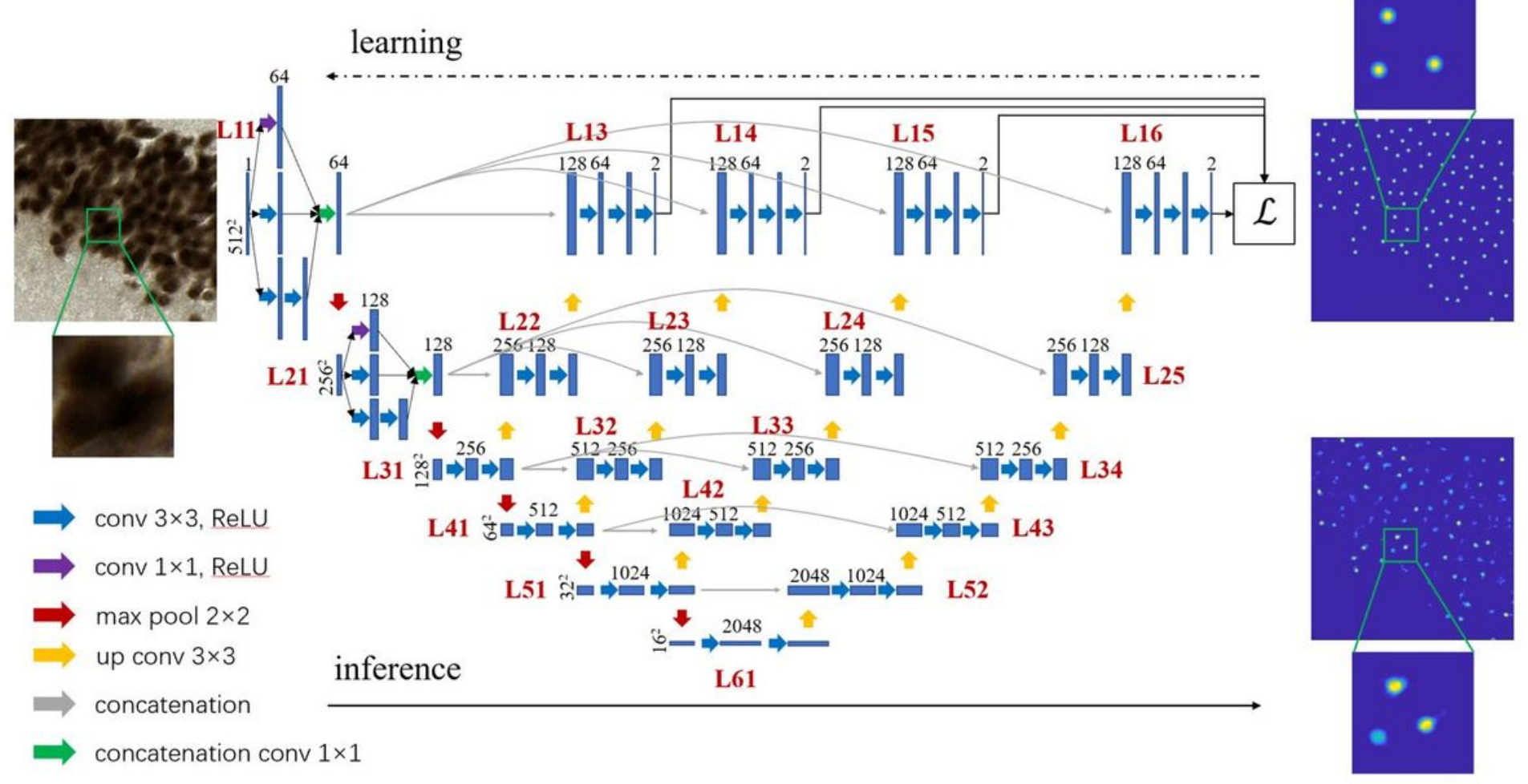

Figure 4

Network structure for neuron centroid detection. The red figures noted as Lxy are the different layers. $x$ represents the layer along the vertical direction and $y$ represents the layer along the horizontal direction. The size of input and output images is $512 \times 512$ pixels. Arrows of different colors represent different operations as described at the left bottom of the figure. The size of images is denoted at the left bottom of the boxes and the number of channels is denoted at the top of the boxes. If there is nothing at the left bottom or on the top, it means the same size of images or the same number of channels as that of previous box. 


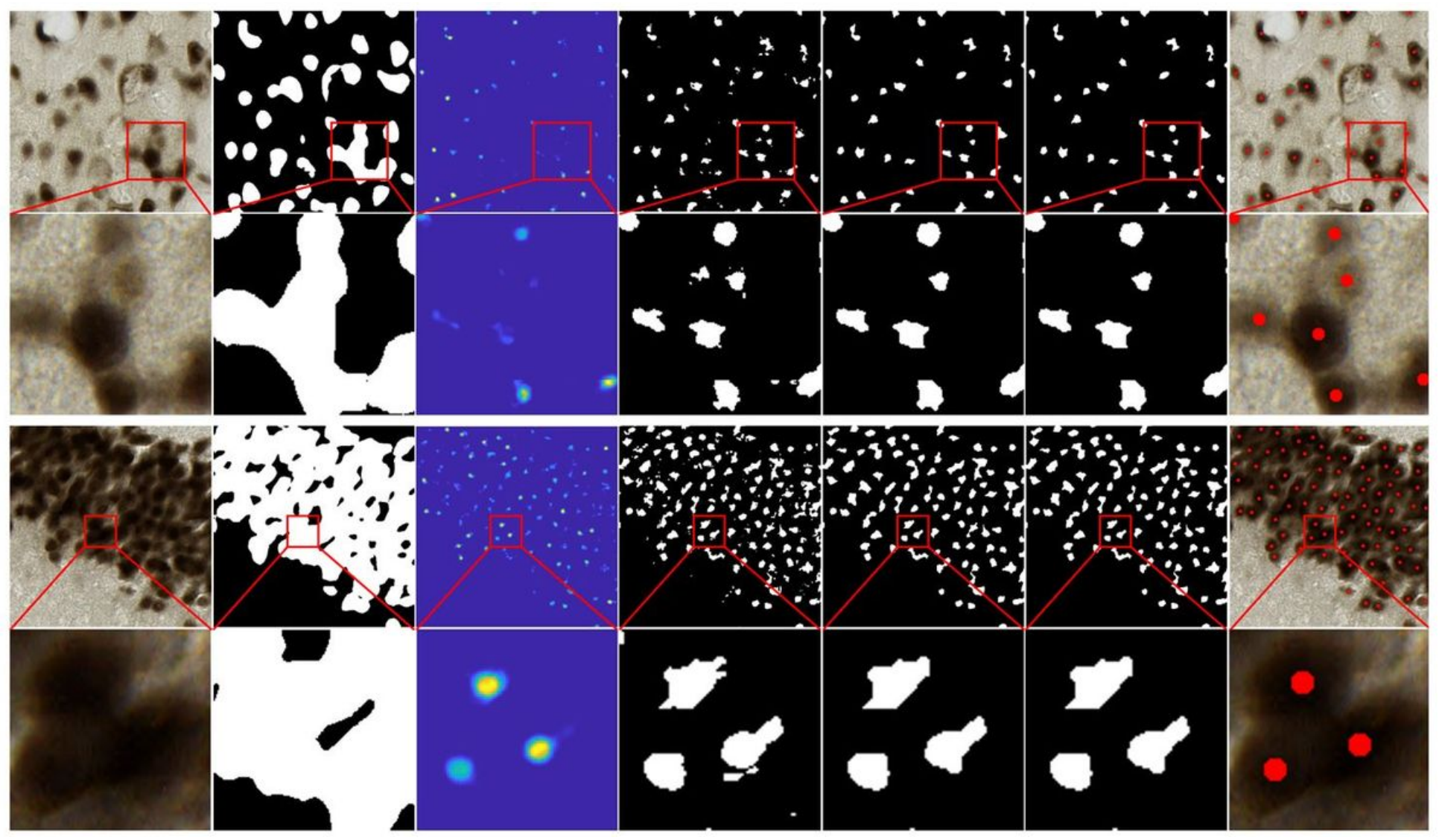

Figure 5

Postprocessing of predicted probability map. a1) \& a2) Example original images of cortex and hippocampus dentate gyrus. b1) \& b2) Neuron mask Im. c1) \& c2) Probability map obtained by using the proposed network. d1) \& d2) Image Ip calculated from c1\& c2) on which the intensity is greater than 0. e1) \& e2) Denoised image based on d1) \& d2) after erosion, reconstruction and closing operations. f1) \& f2) Images of e1) \& e2) masked by Im. g1) \& g2) Original images overlapped with centroids, represented by red disks, calculated as mass center of connected component on f1) \& f2). 


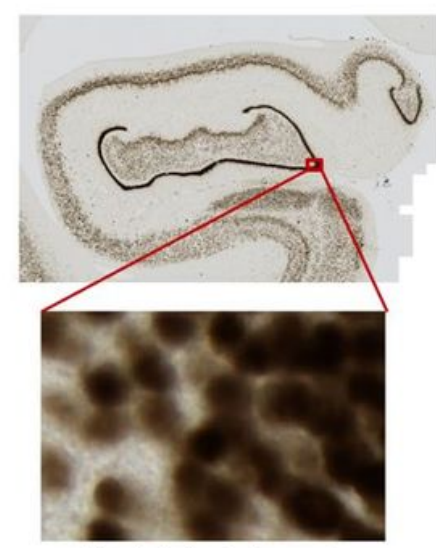

Histological color image of neuronal staining, $2.8 \mathrm{~GB}$

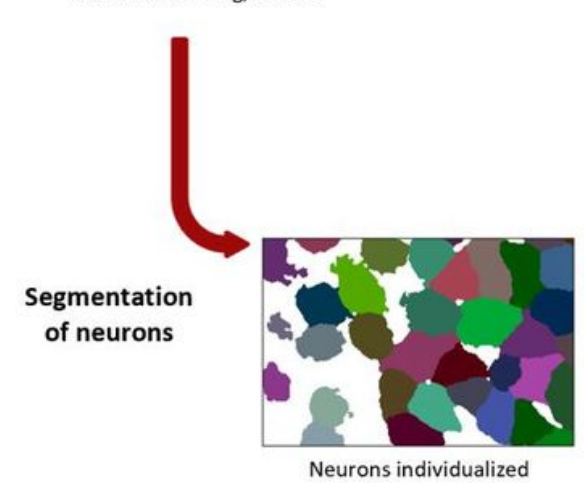

Neurons individualized

\begin{tabular}{|c|c|c|c|c|c|}
\hline Parameters & Cell1 1 & Cell 2 & 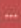 & Cell n & Unit \\
\hline Mass center $x$ & 8 & 19 & -. & 24415 & $\mathrm{px}$ \\
\hline Mass center $y$ & 18176 & 24750 & - & 11782 & $p x$ \\
\hline Area & 439 & 1239 & -. & 1103 & $p x^{2}$ \\
\hline Mean radius & 12.3 & 20.1 & -. & 28.3 & $p x$ \\
\hline Circularity & 0.76 & 0.69 & .. & 0.28 & , \\
\hline Orientation & -88.5 & -51.7 & -. & 69.7 & - \\
\hline$\cdots$ & .. & -. & -. & -. & - \\
\hline Mean red color & 87.7 & 54.9 & .. & 71.8 & , \\
\hline
\end{tabular}

Parameter table (px: pixel; ${ }^{\circ}$ : degree)

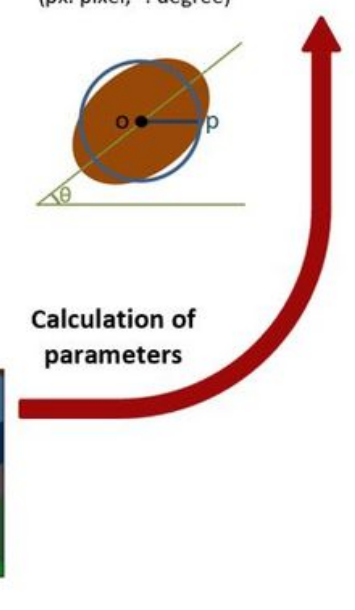

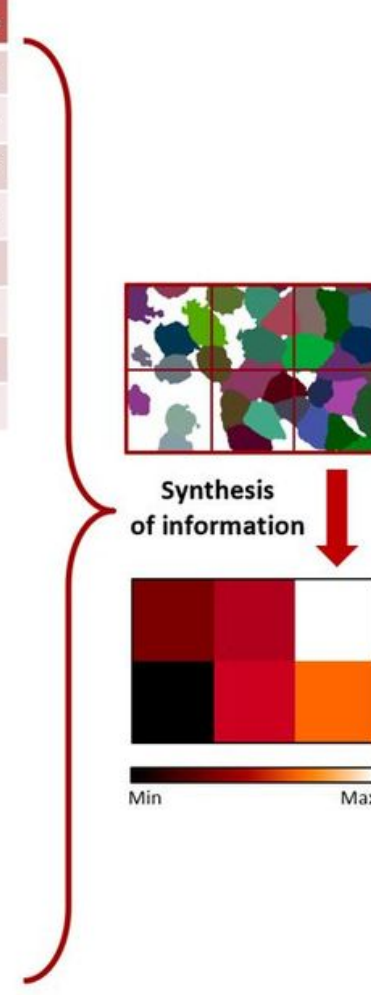

Microscopic $0,22 \mu \mathrm{m} /$ pixel
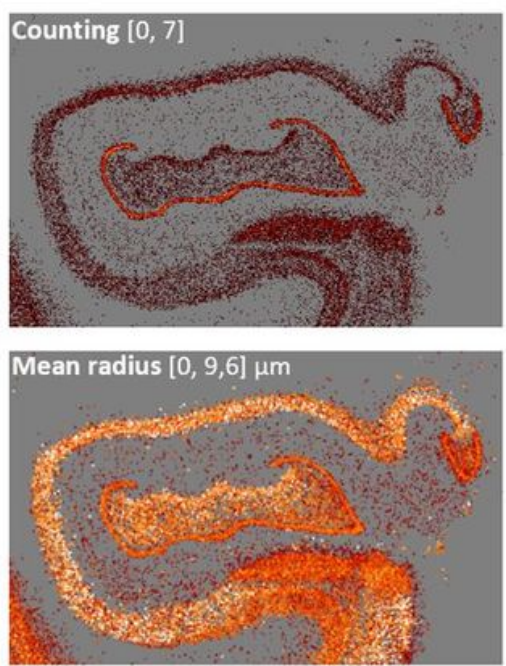

Orientation $\left[-90^{\circ}, 90^{\circ}\right]$

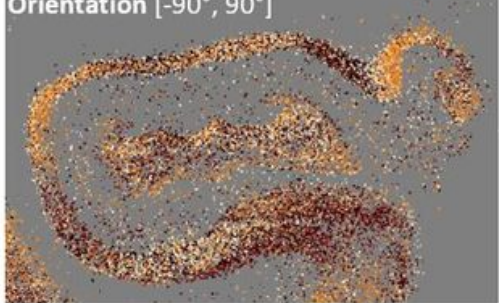

Mesoscopic

$22 \mu \mathrm{m} /$ pixel

Figure 6

Strategy to generate features cartographies of hippocampus at mesoscopic scale. 


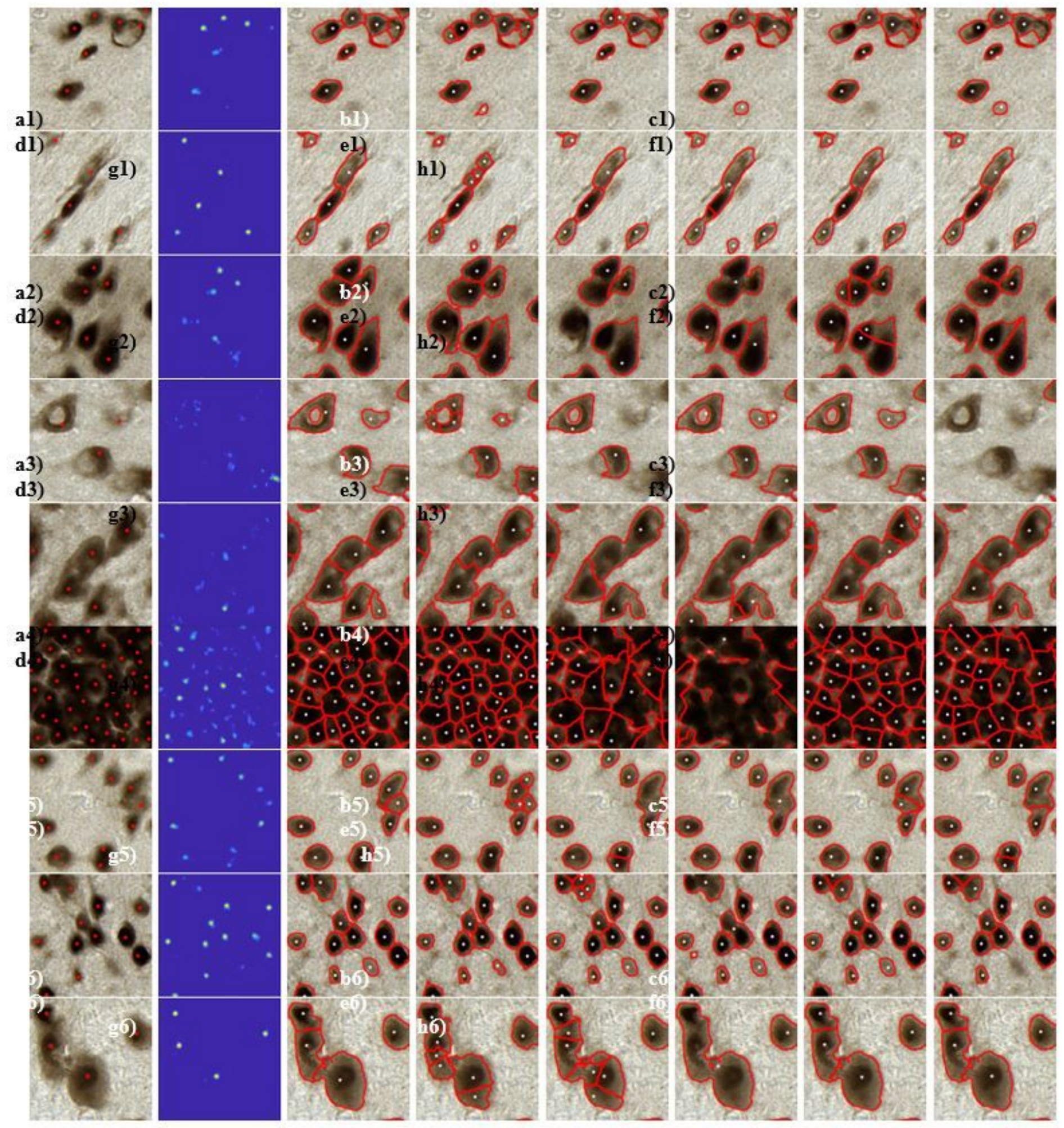

\section{Figure 7}

Comparison results of neuron centroid detection and neuron individualization obtained on images from different anatomical regions. From the 1 st row to the 9 th, the images are from caudate, claustrum, cortex, hippocampus CA1 (cornu ammonis), hippocampus CA3, hippocampus dentate gyrus, putamen, subiculum and thalamus. a1-a9) Original images overlapped with expert annotations marked by red points. b1-b9) Estimated probability map of neuron centroid. c1-c9) Individualization results obtained by 
applying the proposed network. Centroids marked by white points are post-processed based on b1-b9). Red contours are obtained by applying competitive region growing method You et al. (2019). d1-d9) Individualization results obtained based on You et al. (2019). e1-e9) Individualization results obtained by applying U-net (Ronneberger et al., 2015). f1-f9) Individualization results obtained by applying U-net++ (Zhou et al., 2020). g1-g9) Individualization results obtained by applying FCRN (Xie et al., 2016). h1-h9) Individualization results obtained based on You et al. (2020).

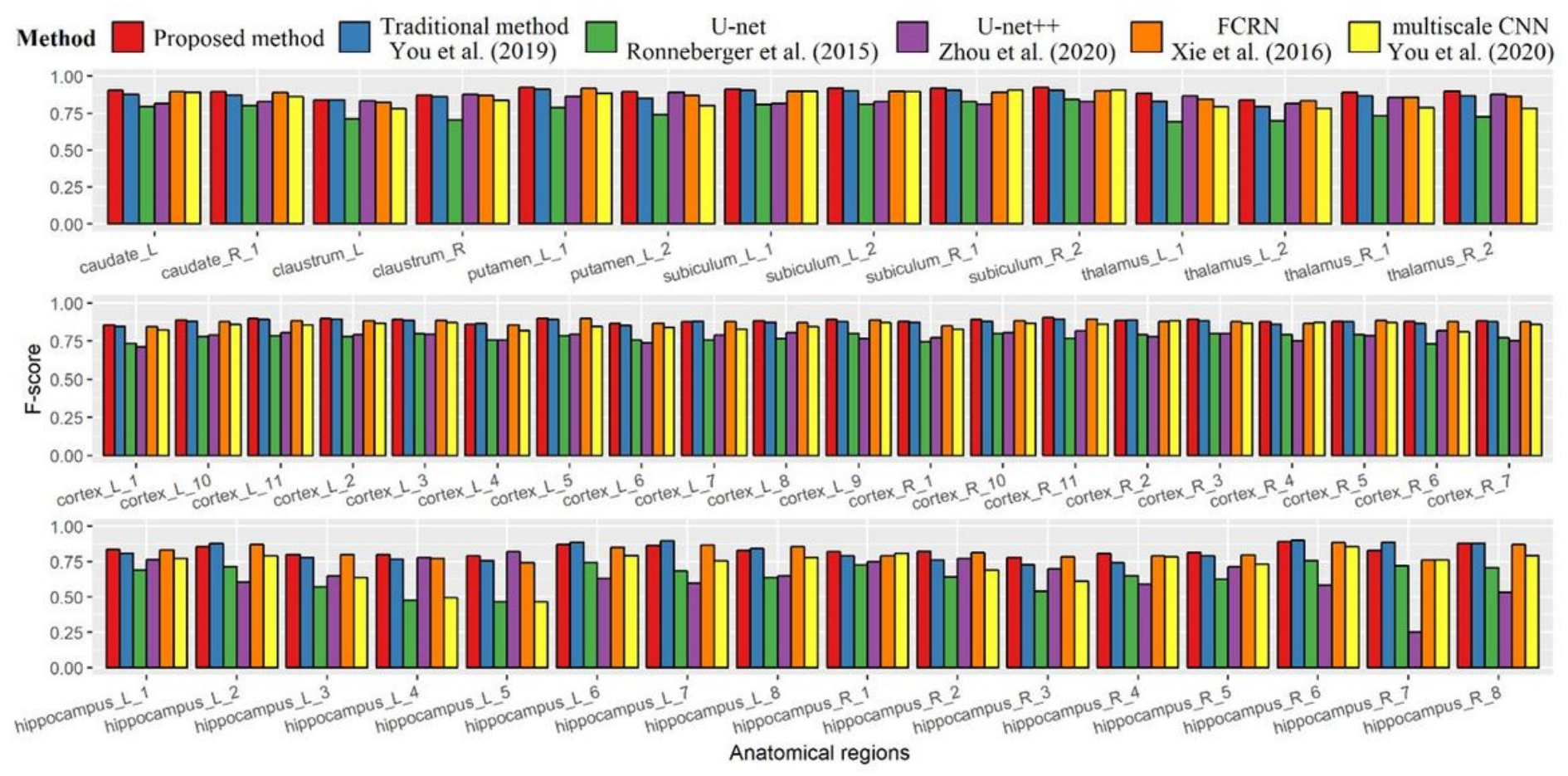

Figure 8

F-score calculated using the proposed method on 50 images of the individualization dataset. $\mathrm{L}$ (respectively, R) means that this image is extracted from the left (respectively, right) side of the section. 


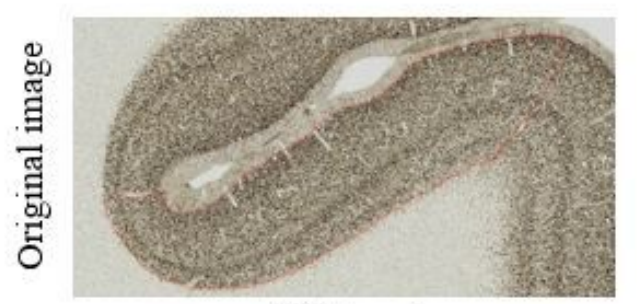

al) S1_cortex

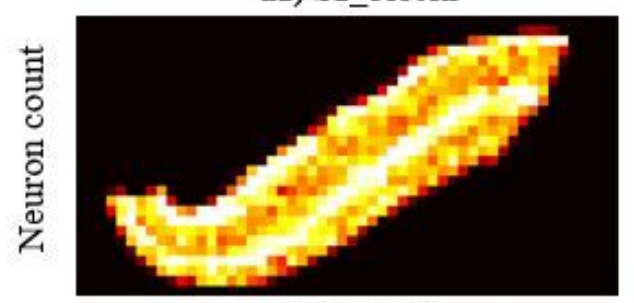

b1) $[0,67]$
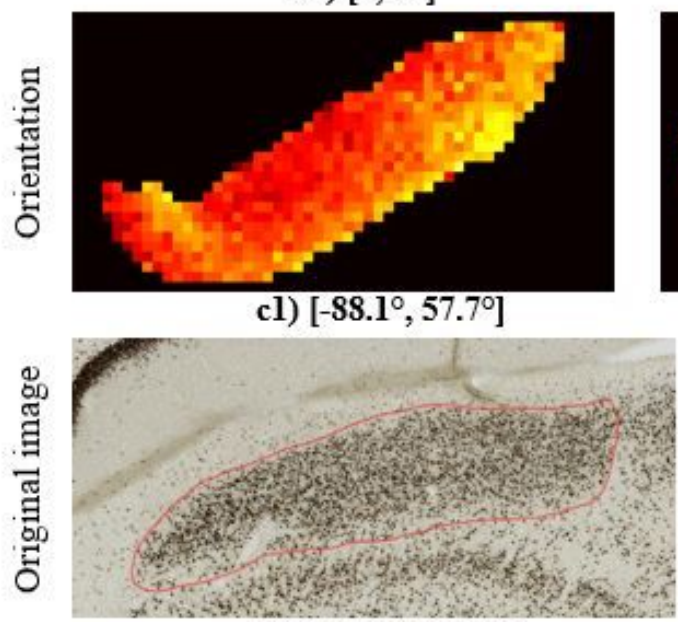

a5) S4_subiculum

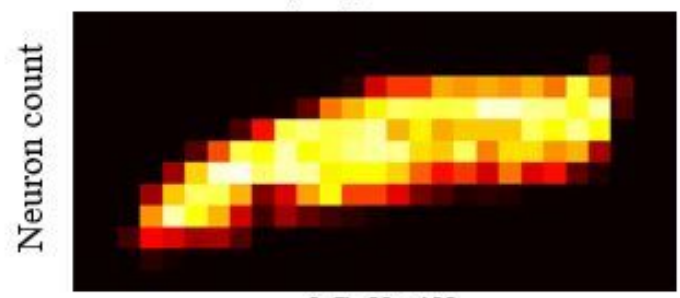

b5) $[0,49]$

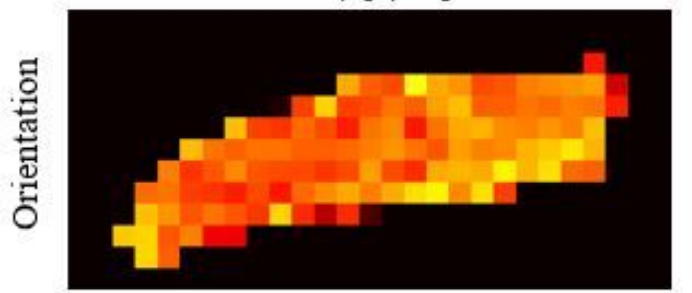

c5) $\left[-82.9^{\circ}, 47.5^{\circ}\right]$

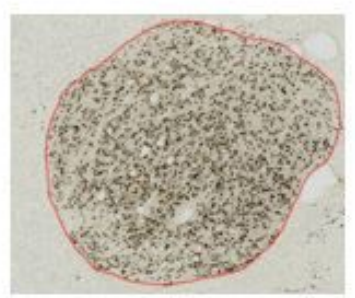

a2) S2_caudate

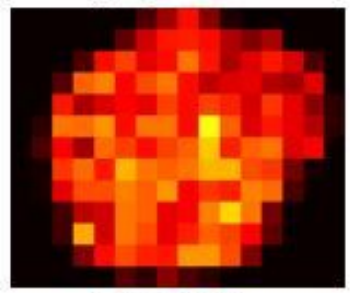

b2) $[0,38]$

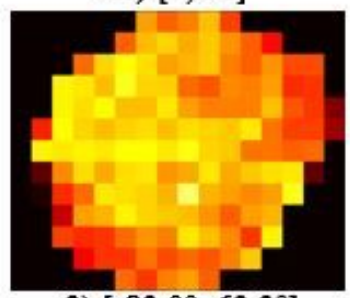

c2) $\left[-83.0^{\circ}, 63.3^{\circ}\right]$

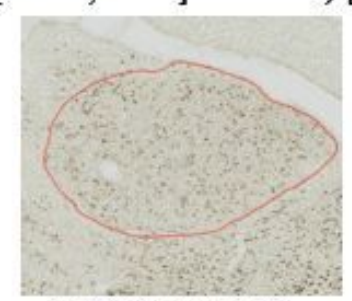

a6) S5_thalamus

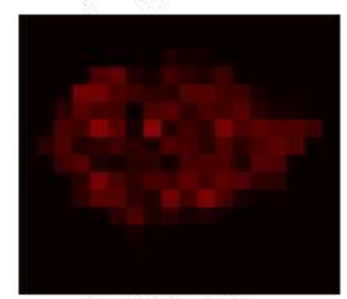

b6) $[0,10]$

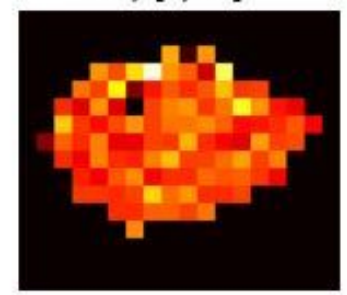

c6) $\left[-73.5^{\circ}, 83.2^{\circ}\right]$ a3) S3 thalamus

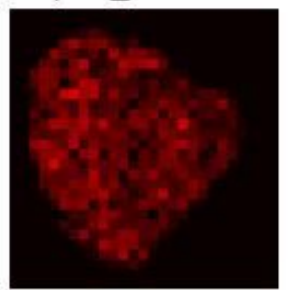

b3) $[0,16]$

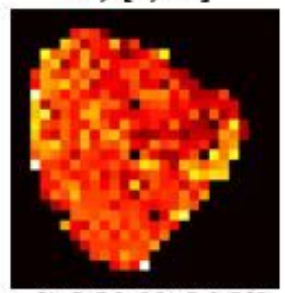

c3) $\left[-83.3^{\circ}, 86.8^{\circ}\right]$

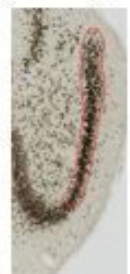

a7) S6_gyrus

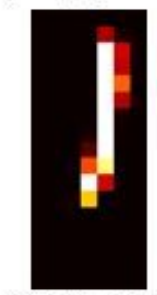

b7) $[0,76]$

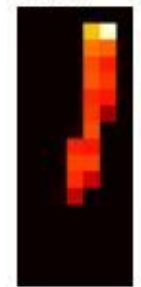

c7) $\left[-86.8^{\circ}, 81.3^{\circ}\right]$

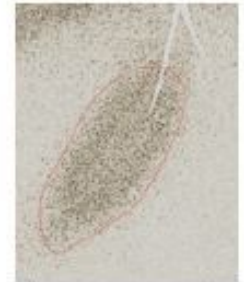

a4) $S 7$ cortex

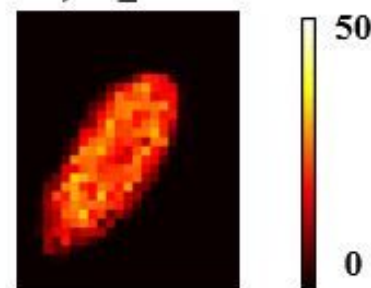

b4) $[0,34]$
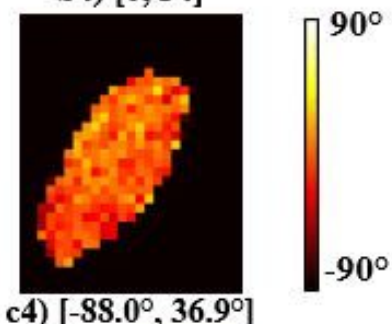

$-90^{\circ}$

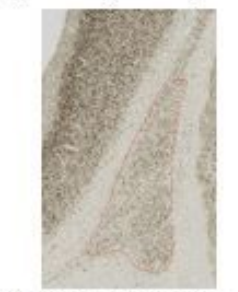

a8) S8_claustrum

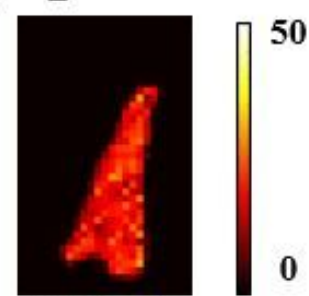

b8) $[0,30]$

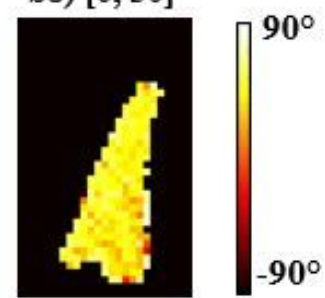

c8) $\left[-31.9^{\circ}, 85.1^{\circ}\right]$

Figure 9

Representation of cartography of two descriptors (the number of neurons and the average of orientation of neurons) in 6 anatomical regions of interest. The aggregation faction is 512 pixels in both horizontal and vertical directions. The resolution at mesoscopic level is $0.11264 \mathrm{~mm} /$ pixel. a1) S1_cortex. a2) S2_caudate. a3) S3_thalamus. a4) S7_cortex. a5) S4_subiculum. a6) S5_thalamus. a7) S6_dentate_gyrus. a8) S8_claustrum. b1-b8) are respectively the cartography of the number of neurons 
and c1-c8) are respectively the cartography of the average of orientation of neurons. The range of the value of descriptors represented by each pixel is listed at right bottom of the image. 